\title{
Eigenhaftung der Organe für die Veranlassung existenzvernichtender Leitungsmaßnahmen im Konzern
}

\author{
Prof. Dr. Tobias Tröger, LL.M. (Harvard) \\ und \\ Stephan Dangelmayer, Frankfurt/München*
}

Inhaltsübersicht

ZGR 2011, 558-588

I. Ungeklärte Reichweite von Bestandsverantwortung und Sonderdeliktshaftung im Unternehmensverbund . . . . . . . . . . . . . . . . . . . . . .

II. Entwicklung des Haftungskonzepts . . . . . . . . . . . . . . . . . . 563

1. Verlustausgleichspflicht im „qualifizierten faktischen Konzern“ . . . . . 564

2. Verlustausgleichspflicht als Folge einer Durchgriffshaftung bei missbräuchlichem Verhalten . . . . . . . . . . . . . . . . . . . 565

3. Deliktische Verhaltenshaftung der Bestandsverantwortlichen . . . . . . 566

III. Legitimation der Verantwortlichkeit auf der Grundlage bestehender PrinzipalAgenten-Beziehungen . . . . . . . . . . . . . . . . . 568

IV. Konzernspezifische, fiduziarische Bindungen der Leitungsorgane im deutschen Gesellschaftsrecht . . . . . . . . . . . . . . . .

1. Leitungskompetenz und -verantwortung im Konzern . . . . . . . . . . .

2. Das abweichende Haftungskonzept nach $\$ \int 309$ Abs. 2, 317 Abs. 3 AktG und die Parallelen im GmbH-Konzernrecht . . . . . . . . . . . . . . .

V. Konsequenzen für die Passivlegitimation bei der Existenzvernichtungshaftung

1. Scheinlösung über eine Binnenhaftung nach $\$ 823$ Abs. 2 BGB i. V.m. $\$ 266$

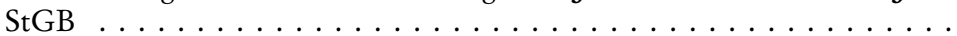

2. Scheinlösung über die Haftung als Tatteilnehmer, $\$ 830$ Abs. 1, Abs. 2 BGB

3. Vorzugswürdigkeit des Fortschreibens der gesellschaftsrechtlichen Verant-

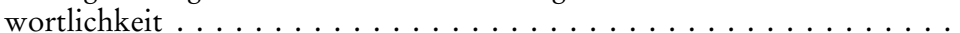

VI. Übertragbarkeit auf den Aktienkonzern . . . . . . . . . . . . . . . . . . . . . 585

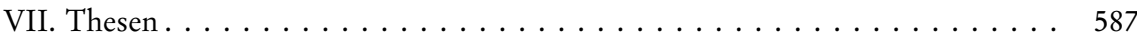

Mit der "Trihotel“-Entscheidung hat der 2. Zivilsenat des Bundesgerichtshofs das Haftungskonzept bei „existenzvernichtenden Eingriffen"im Jabr 2007 in der qualifizierten deliktsrechtlichen Generalklausel des $\int 826$ BGB verankert. Mit dieser positiv rechtlichen Anknüpfung scheint auch die einst im Zentrum des „Bremer Vulkan“-Verfabrens stehende - und in der juristischen Aufarbeitung der nach Deutschland reichenden Ausläufer der Finanzkrise

* Prof. Dr. Tobias Tröger, LL.M. (Havard) ist Inhaber der Professur für Bürgerliches Recht, Handels- und Wirtschaftsrecht an der Goethe Universität Frankfurt am Main. Stephan Dangelmayer ist Doktorand und Wissenschaftlicher Mitarbeiter am Lehrstuhl für Bürgerliches Recht und Unternehmensrecht (Prof. Dr. Mathias Habersack) der Ludwig-Maximilians-Universität München. 
zunebmend an Bedeutung gewinnende - Frage nach der Haftung auch des Konzernvorstands für Maßnabmen, die primär eine Insolvenz der Tochtergesellschaft verursachen, affirmativ beantwortet zu sein. Schließlich stellt sich der Kreis der potentiell Passivlegitimierten bei einer vorsätzlichen sittenwidrigen Schädigung im Ausgangspunkt als unbegrenzt dar. Ein simpler Schluss von der Rechtsnatur deliktischer Jedermannsplichten auf die Reichweite der in Rede stehenden Sonderdeliktshaftung ist gleichwobl verfehlt. Der Beitrag unternimmt die notwendige normative Absicherung der Ergebnisse, die auf der Grundlage genuin verbandsrechtlicher Wertungen, im Rabmen der Dogmatik des Organisationsrechts erfolgen muss. Auf der Grundlage institutionenökonomischer Einsichten wird gezeigt, dass eine Verantwortlichkeit der Verwaltungsmitglieder der Obergesellschaft prinzipiell sachgerecht ist. Eine bloße Binnenhaftung der Organwalter der Obergesellschaft gegenüber Ibrer Anstellungskörperschaft erweist sich aufgrund von Vollzugsdefiziten als unzureichend. Die zivilrechtlich durch eine Außenhaftung sanktionierte Bestandsverantwortung auch der Leitungsorgane der Obergesellschaft ist die Konsequenz des in $\iint 309$ Abs. 2, 317 Abs. 3 AktG zum Ausdruck gebrachten Gleichlaufs von Haftungsverantwortung und konzerndimensionaler Leitungsmacht. Dem entspricht im Rabmen der Tribotel-Doktrin allein die Annabme einer eigenen Pflicht der Verwaltungsmitglieder, deren vorsätzliche Verletzung unmittelbar zur Haftung nach $\int 826 B G B$ führt.

In 2007 the 2nd civil-panel of the German Federal Court of Justice („Bundesgerichtshof“) rendered its Tribotel-judgment. The Court held that the doctrinal basis of the well-established shareholder-liability for imperiling a limited liability corporation's subsistence („Existenzvernichtungshaftung ") is situated in general tort law. As a consequence, it seems that the Court has affirmed the much-disputed personal liability of the parent corporation's directors if they willfully cause a subsidiary's bankruptcy. The question not only lay at the heart of the Court's famous Bremer Vulkan-judgment but also has become incrementally important where the ramifications of the global financial crisis reach Germany and suggest legal consequences for certain corporate agents. After all, the circle of persons that potentially can be beld liable under $\int 826$ of the German Civil Code („Bürgerliches Gesetzbuch“) for willfully and unconscionably causing harm to another is unlimited at the outset. However, it would be misguided to infer form the pertinent liability rule's legal nature as a general tort sanction that its specific occurence in corporate law attaches to any corporate agent. This article undertakes the necessary normative underpinning of the appropriate results. It thereby dwells upon genuine principles of corporate law and aligns the conclusions with the prevalent doctrine of organizational law. The insights of New Institutional Economics, particularly the theory of incomplete contracts indicate that the accountability of board-members of the parent company is generally adequate and desirable. The internal liability of board-members vis-à-vis the corporation that employs them proves insufficient in light of substantial shortcomings in the pertinent rules' enforcement. The responsibility for the subsidiary's economic viability also concerns the board members of the parent corporation. Their liability to the subsidiary is a consequence of the synchronism between private law accountability and administrative power in a corporate group as expressed in $\iint 309$ (2), 317 (3) of the German Stock Corporation Code ("Aktiengesetz"). In line with the Tribotel-doctrine, these considerations mandate an innate obligation of the parent's board members. Its willful breach leads immediately to their liability under $\int 826$ of the German Civil Code. 


\section{Ungeklärte Reichweite von Bestandsverantwortung und Sonderdelikts- haftung im Unternehmensverbund}

Die vor kurzem mit unspektakulären Vergleichen für einige der beteiligten Vorstände abgeschlossene privatrechtliche Aufarbeitung der Vorgänge um die Insolvenz der Bremer Vulkanwerft ${ }^{1}$ hat in einem früheren Stadium zu einer fundamentalen Neuorientierung des dogmatischen Konzepts der Verantwortlichkeit für bestandsgefährdende Leitungsmaßnahmen im Gesellschaftsrecht geführt. ${ }^{2}$ Die dabei ursprünglich eingeschlagene Linie ist allerdings schon 2007 wieder verlassen worden, als das positivrechtliche Fundament der Judikatur ein weiteres Mal ausgetauscht wurde. ${ }^{3}$ Dernière crie dieser mäandernden Rechtsprechung ist nunmehr die qualifizierte deliktsrechtliche Generalklausel des $\$ 826$ BGB, die dazu dient, die Haftung des Veranlassers von „existenzvernichtenden Eingriffen " in der lex lata zu verankern. Damit scheint die ursprünglich im Zentrum des Bremer Vulkan-Verfahrens ${ }^{4}$ stehende Frage nach der Haftung auch des Konzernvorstands für Maßnahmen, die primär eine Insolvenz der Tochtergesellschaft verursachen, im affirmativen Sinne beantwortet zu sein. Schließlich ist der Kreis der Passivlegitimierten, die für eine vorsätzlich sittenwidrige Schädigung Anderer einstehen müssen, im Ausgangspunkt unbegrenzt und erfasst daher vermeintlich problemlos auch den leitungsmächtigen Konzernvorstand. ${ }^{5}$

Eine kurze Besinnung auf die dogmatische Konzeption des $\$ 826$ BGB als Haftungsbestimmung, deren Ziel schon aus der Fassung des Tatbestands er-

1 Die Welt Nr. 57 v. 9.3.2010, S.33.

2 BGHZ 149, 10; in (vermeintlicher) Fortführung des dort eingeschlagenen Weges, wenn auch z. T. mit erkennbaren Absetzungstendenzen z. B. BGHZ 151, 181 „KBV“; BGH ZIP 2005, 117; BGH ZIP 2005, 250; BGH ZIP 2005, 1734.

3 BGHZ 173, 243; bekräftigt in BGH ZIP 2008, 308; BGH ZIP 2008, 1329; BGH ZIP 2009, 802.

4 Die Klage richtete sich - wegen der die gesamte Werften-Gruppe erfassenden Insolvenzgegen die Vorstände der Konzernholding, vgl. BGHZ 149, 10, 11.

5 Entsprechend wird in der Literatur konstatiert, die Haftung der Organe des herrschenden Unternehmens bereite unter dem „neuen Haftungsmodell“ nach \826 BGB „keinerlei Schwierigkeiten mehr“, Liebscher, Münchener Komm. z. GmbHG, Bd. 1, 2010, Anh. $\$ 13$ Rdn. 591. In der Bremer Vulkan-Entscheidung hielt der BGH demgegenüber eine Haftung der Vorstände der Gesellschafterin auf der Grundlage des Verbots bestandsvernichtender Eingriffe für „nicht begründbar“, BGHZ 149, 10, 17. Im Ergebnis für Haftung des Vorstands jetzt auch EmMERIcH, in: Emmerich/Habersack, Aktien- und GmbH-Konzernrecht, 6. Aufl., 2010, \$309 Rdn. 53; HaAs in: Baumbach/Hueck, Komm. z. GmbHG, 19. Aufl., 2010, \$64 Rdn. 176; HaAs, ZIP 2006, 1373, 1380 f; Wagner, FS Claus-Wilhelm Canaris, 2007, S. 473, 496; vgl. auch DERs., Münchener Komm. z. BGB, Bd. 5, 5. Aufl., 2009, $\ 826$ Rdn. 36; über eine Teilnehmerhaftung der Organwalter gemäß \830 Abs. 2 BGB Kölbl, BB 2009, 1194, 1198; LutTER, in: Lutter/Hommelhoff, GmbHG, 17. Aufl., 2009, $\$ 13$ Rdn. 46; Altmeppen, in: Roth/Altmeppen, GmbHG, 6. Aufl., 2009, $\mathbb{1 3}$ Rdn. 102; VetTer, BB 2007, 1965, $1968 \mathrm{f}$. 
sichtlich die Sanktionierung von qualifiziertem Verhaltensunrecht ist, ${ }^{6}$ lässt aber erkennen, dass die unreflektierte Annahme einer konzernweiten Bestandsverantwortung auch der Leitungsorgane der Obergesellschaft als zwangsläufiger Ausfluss einer deliktischen Jedermannspflicht fehl ginge. Vielmehr muss ein solcher Imperativ, dessen vorsätzliche Verletzung zur Grundlage einer Haftung nach $\ 826$ BGB gemacht werden kann, für sämtliche in Pflicht genommenen Akteure sowohl hinsichtlich seiner Existenz als auch seiner Reichweite aus dem teleologischen Kontext des einschlägigen Organisationsrechts begründet werden. Mit anderen Worten, trotz der Verortung der Haftung im allgemeinen Deliktsrecht verliert diese ihre genuin verbandsrechtliche Prägung nicht. ${ }^{7}$ In der Konsequenz ist anhand originär gesellschaftsrechtlicher Wertungskriterien zu entscheiden, welche Beteiligten eine, im Falle ihrer Missachtung zu sanktionierende Bestandsverantwortung für die betroffene Gesellschaft trifft und wie weit diese reicht.

Die skizzierten Zusammenhänge werden in der Literatur durchaus gesehen, wenn die Haftung nach Trihotel als „Sonderdeliktshaftung" charakterisiert wird, ${ }^{8}$ die nur von solchen Personen verwirklicht werden könne, die eine Bestandsverantwortung für die Gesellschaft tragen. ${ }^{9}$ Ob der Kreis der in diesem Sinne in die Pflicht Genommenen zutreffend umschrieben wird, wenn ausschließlich die Alleingesellschafter bzw. die im Konsens agierenden Anteilseiger als potentielle Haftungsadressaten genannt werden, ${ }^{10}$ erscheint al-

6 Im Rahmen der qualifizierten Generalklausel des $₫ 826$ BGB taugt die kausale Verursachung des tatbestandlichen Schädigungserfolgs unstreitig nicht als objektive Grundlage der Legitimation der Überwälzung der eingetretenen Einbußen. Insoweit besteht in der Literatur Übereinstimmung, obwohl i.Ü. das Verhältnis von Sittenverstoß und Vorsatzerfordernis bei der Vermessung des Normzwecks kontrovers ist, z. B. WAGNER, aaO (Fn. 5) $\$ 826$ BGB Rdn. 3; Oechsler, in: Staudinger, BGB, Neubearb. 2009, $\ 826$ Rdn. 15; Schiemann, in: Erman, BGB, 12. Aufl., 2008, $\$ 826$ Rdn. 2; Spindler, in: Bamberger/Roth, BGB, Bd.2, 2. Aufl., 2007, $\mathbb{8 2 6}$ Rdn.1. Der v. a. im Hinblick auf \823 Abs. 1 BGB fundamentale Streit zwischen der Lehre vom Erfolgs- und derjenigen vom Verhaltensunrecht kommt insoweit daher nicht zum Tragen, dazu nur Hager, in: Staudinger, BGB, Neubearb. 2009, \823 Rdn. H 14 ff; Larenz/Canaris, Lehrbuch des Schuldrechts II/2, 13. Aufl., 1994, $\$ 75$ II. 3., S. 364 ff; KöTZ/WaGner, Deliktsrecht, 10. Aufl., 2006, Rdn. $102 \mathrm{ff}$.

7 WaGner, FS Claus-Wilhelm Canaris, S. 473, $492 \mathrm{ff}$.

8 Eingehend zuletzt S. Schneider, FS Uwe H. Schneider, 2011, S. 1177, $1180 \mathrm{ff}$; für die h. M. Habersack, in: Emmerich/Habersack, Aktien- und GmbH-Konzernrecht, 6. Aufl., 2010, Anh. zu $\$ 318$ Rdn.39; Altmeppen, aaO (Fn.5), $\$ 13$ GmbHG Rdn. $101 \mathrm{ff}$ \433 GmbHG Rdn. 86; Weller, ZIP 2007, 1681, 1687; Schaefer/STEINMETZ, WM 2007, 2265, 2271; GeHrLein, WM 2008, 761, 764; Habersack, ZGR 2008, 533, 546; HönN, WM 2008, 769, 770; WITt, DNotZ 2008, 220, 225.

9 BGHZ 173, 246 Rdn. 25; dazu auch Weller, ZIP 2007, 1681, 1689; bereits vor „Trihotel“ Wagner, FS Claus-Wilhelm Canaris, S. 473, 479 f, 495 f, der freilich davon ausgeht, die Haftung beruhe nicht auf einem Sonderdelikt (ibid., S. 496).

10 In diesem Sinne z. B. Habersack, aaO (Fn. 8), Anh. zu \318 Rdn. 39; wohl auch Zöll- 
lerdings zweifelhaft. Zwar haften die Mitglieder des Leitungsorgans für die Vernichtung der Existenz „ihrer“ Gesellschaft ohne Weiteres aus $\$ 93$ Abs. 2 Satz 1 AktG, $\$ 43$ Abs. 2 GmbHG als Ausfluss ihrer allgemeinen Organpflichten - zweifelhaft ist allenfalls die Reichweite ihrer Bestandsverantwortung. ${ }^{11}$ Im geltenden Recht weniger klar verortet ist indessen die Antwort auf die Frage, ob auch die Geschäftsleiter einer Konzernobergesellschaft eine Verantwortlichkeit für den Bestand untergeordneter Verbundgesellschaften trifft, die haftungsrechtlich unmittelbar durch einen Kompensationsanspruch der betroffenen Tochtergesellschaft sanktioniert wird. ${ }^{2}$ Dies gilt jedenfalls soweit keine Nachteilszufügung dargetan werden kann, die im Rahmen des auf isolierbare Einzelschädigungen abstellenden Systems der $\$ 311 \mathrm{ff} \mathrm{AktG}$ gemäß $\mathbb{S} 3317$ Abs. 3 AktG zur Eigenhaftung der Handelnden führen könnte. ${ }^{13} \mathrm{Un}-$ klar erscheint vor diesem Hintergrund, ob die zu konstatierende Enthaltsamkeit der einschlägigen Gesetze einer nachvollziehbaren teleologischen Konzeption entspringt, oder aber eine Schutzlücke signalisiert, die nicht zuletzt durch Rechtsfortbildungen à la Existenzvernichtungshaftung geschlossen werden muss. ${ }^{14}$

Die Finanzkrise zeigt die Brisanz der vorgezeichneten Fragestellung: Jenseits der durch Alleingesellschafter oder abgestimmt agierende Eignergemeinschaften dominierten Kleinstunternehmen stellt sich rasch die Frage, ${ }^{15}$ welche Bestandsrisiken die Leitungsorgane eines Unternehmensverbunds zu Lasten der

NER, in: Baumbach/Hueck, Komm. z. GmbHG, 19. Aufl., 2010, SchlAnhKonzernR Rdn. $124 \mathrm{f}$.

11 Die Relevanz der letzten Frage soll dabei nicht heruntergespielt werden. Die als Anfechtung der Vorstandsentlastung eingekleidete, in Kompetenzfragen verhaftete Entscheidung des LG Frankfurt, BB 2010, 980 und noch deutlicher die Berufungsentscheidung OLG Frankfurt, WM 2011, 116 in Sachen Commerzbank/Dresdner Bank zeigen, ebenso wie das Verfahren um die Vorgänge bei der IKB (dazu unten Fn. 16), dass die exakte Bestimmung der Verantwortlichkeit über Haftungsrisiken in Milliardenhöhe entscheiden kann. Eingehend dazu Baums, ZGR 2011, 218 ff.

12 Dafür, mit abweichenden dogmatischen Begründungen die in Fn. 5 Genannten.

13 Die aktienrechtliche Regelung zum faktischen Konzern und damit auch der Haftungserstreckung in $₫ 317$ Abs. 3 AktG wird bei nicht in der Rechtsform der AG organisierten Untergesellschaften überwiegend für unanwendbar gehalten vgl. bereits BGHZ 95, 330, 340; Zöllner, aaO (Fn. 10), SchlAnhKonzernR Rdn. 127; Lutter, aaO (Fn. 5), Anh. $\mathrm{Zu} \ 13 \mathrm{GmbHG}$ Rdn. 20 (zur GmbH); Hopt, in: Baumbach/Hopt, HGB, 34. Aufl., 2010, \$105 Rdn. 103; Emmerich/Habersack, Konzernrecht, 9. Aufl., 2008, \$34 Rdn. 3 (für die Personenhandelsgesellschaften). Gerade im Hinblick auf die Haftungserstreckung des $₫ 317$ Abs. 3 AktG im Personengesellschaftsrecht befürwortend aber MüLBert, Münchener Komm. z. HGB, 2. Aufl., 2007, KonzernR, Rdn. 212; U. Schneider, BB 1980, 1057, 1065.

14 Im Ausgangspunkt ebenso, wenn auch auf die Teilnehmerhaftung nach $\$ 830$ Abs. 2 BGB verengt, jetzt S. Schneider, FS Uwe H. Schneider, 2011, S. 1177, 1188, 1191.

15 Ähnliche Einschätzung der Praxisrelevanz v. a. mit Blick auf die mittelständische Wirtschaft bei S. SchneIDER, FS Uwe H. Schneider, 2011, S. 1177, $1178 \mathrm{f}$. 
Gläubiger und ggf. der Minderheitsaktionäre von Konzerngliedern, aber auch solcher der Verbundspitze, ${ }^{16}$ eingehen dürfen und wo diese den Rubikon überschreiten und daher haftungsrechtlich im Interesse konzernfreier Residualgläubiger und der Fremdkapitalgeber in die Pflicht zu nehmen sind.

Der vorliegende Beitrag versucht eine normative und dogmatische Grundlegung für die aufgeworfenen Fragen zu schaffen. Zu diesem Zweck werden kurz die dogmatischen Konzeptionen der rechtsförmig sanktionierten Bestandsverantwortung in Erinnerung gerufen, soweit sie im Rahmen der wechselhaften Entwicklung der Existenzvernichtungshaftung über die Inpflichtnahme der gesetzlichen Vertreter der Obergesellschaft bestimmend waren. ${ }^{77}$ Vor diesem Hintergrund wird mit Hilfe institutionenökonomischer Überlegungen das Bedürfnis nach einer konzerndimensionalen Pflichtenbindung eben dieser Organe belegt, ${ }^{18}$ um auf dieser Grundlage die Tauglichkeit der Rechtsinstitute zu ventilieren, die diesem Bedürfnis im deutschen Verbandsrecht Rechnung tragen könnten. ${ }^{19}$ Die hierbei zu konstatierenden Unzulänglichkeiten liefern die Legitimation für eine Erstreckung der durch $\$ 826$ BGB sanktionierten Bestandsverantwortung auch auf die gesetzlichen Vertreter des herrschenden Unternehmens. ${ }^{20}$ Dabei kann gezeigt werden, dass die primär für die Einpersonen-GmbH entwickelten Grundsätze auch in der beherrschten Aktiengesellschaft Geltung beanspruchen. ${ }^{21}$

\section{Entwicklung des Haftungskonzepts}

Die „Geschichte“ der Existenzvernichtungshaftung und ihres Vorfahren, dem qualifizierten faktischen Konzern, gehört zu den vielgesungenen Bänkelliedern über die Taten des II.Zivilsenats, weshalb einige Hinweise auf die Eckpunkte genügen, soweit diese für die hier untersuchte Frage relevant sind. ${ }^{22}$

16 Eine erste Annäherung an die Aufarbeitung insoweit erfolgt im Zusammenhang mit den Vorgängen um die IKB, vgl. OLG Düsseldorf, ZIP 2010, 28, die, jenseits der dogmatischen Scheinkontroversen, ihren Dreh- und Angelpunkt in eben jener Frage nach der Reichweite der Bestandsverantwortlichkeit der Leitungsorgane findet. Dazu auch Florstedt, AG 2010, 315; Fleischer, NJW 2010, 1504; Spindler NZG 2010, 281.

17 S. u. II.

18 S.u. III.

19 S.u. IV.

20 S. u. V.

21 S. u. V.3.c), VI.

22 Eingehende Analyse von Rechtsprechung und Literatur bei Liebscher, aaO (Fn. 5), Anh. $\$ 13 \mathrm{GmbHG}$ Rdn. $475 \mathrm{ff}$. 


\section{Verlustausgleichspflicht im "qualifizierten faktischen Konzern"}

Mit der Entscheidung „Autokran“23 legte der BGH 1985 den Grundstein für eine besondere Haftung im qualifizierten faktischen GmbH-Konzern. ${ }^{24}$ In analoger Anwendung der für den Vertragskonzern geltenden Regelungen, namentlich $\$ \int 303,322 \mathrm{AktG}$, sollte eine herrschende Gesellschaft für sämtliche Verbindlichkeiten einer abhängigen, vermögenslosen $\mathrm{GmbH}$ einzustehen haben. Aus der hier interessierenden Perspektive ist weniger relevant, dass der BGH zunächst sein Haftungsmodell einer Konzernstrukturhaftung weiter entwickelte („Tiefbau“"25, „Video“"26), um in der Entscheidung „TBB“ ${ }^{27}$ klare Absetzungstendenzen in Richtung auf eine pflichtenbasierte Verhaltenshaftung erkennen zu lassen. Entscheidend ist allein, dass die Verankerung der Verantwortlichkeit in der Analogie zu den $\$ \$ 302,303$ AktG zu einer umfassenden, quasi-akzessorischen Einstandspflicht für sämtliche Schulden der Untergesellschaft führte, die geschädigte Gläubiger von einem Nachweis der Kausalität der Leitungstätigkeit für ihre Einbußen entband.

Dieser Rückgriff auf Regelungen des Konzernrechts blieb gerade für die Haftung der Leitungsorgane der Obergesellschaft nicht ohne Konsequenz. Verletzte das Organ der herrschenden Gesellschaft eine seinem Unternehmen gegenüber der Konzerntochter obliegende Pflicht, so war seine Schadensersatzpflicht auf der Grundlage der Haftungserstreckung des $\$ 309$ Abs. 2 AktG zumindest in Erwägung zu ziehen..$^{28}$ Die umfassende Verlustausgleichspflicht der $\$ \$ 302,303 \mathrm{AktG}$ war auf diesem Weg freilich nicht ausdehnungsfähig, da die Haftung nach $\$ 309$ Abs. 2 Satz 1 AktG ja einen auf eine konkrete

23 BGHZ 95, 330, 341 ff; zust. Emmerich, GmbHR 1987, 213 ff; Priester, ZIP 1986, 137 ff; K. Sснмidt, BB 1985, 2074 ff; Stimpel, AG 1986, 117 ff; Ulmer, AG 1986, 123 ff; krit. Wilhelm, DB 1986, $2113 \mathrm{ff}$.

24 Vorgespurt in Hueck/LutTer/Mertens/Rehbinder/Ulmer/Wiedemann/ZöLlner, Arbeitskreis GmbH-Reform, Bd. 2, 1972, S. 49 ff.

25 BGHZ 107, 7 ff; zust. Emmerich, JuS 1990, 329; K. Schmidt, ZIP 1989, 545; Stimpel, ZGR 1991, 144.

26 BGHZ 115, $187 \mathrm{ff}$, das im Gegensatz zu den vorigen Entscheidungen überwiegen kritisch aufgenommen wurde, vgl. Altmeppen, DB 1991, 2225 ff; Flume, ZIP 1992, 817 ff; K. Schmidt, ZIP 1991, 1325 ff; Westermann, DWiR 1992, $197 \mathrm{ff}$.

27 BGHZ 122, 123 ff; zust. Goette, DStR 1993, 568 ff; Hommelhoff, ZGR 1994, 395 ff; LutTer, JZ 1993, $580 \mathrm{ff}$; im Grundsatz auch Westermann, ZIP 1993, 554 ff; krit. K. SCHMidt, AG 1994, $189 \mathrm{ff}$.

28 Habersack, aaO (Fn. 8), 2. Aufl., 2001, Anh. II zu $\$ 318$ Rdn. 30. Für Haftung der Leitungsorgane der herrschenden Gesellschaft analog $\$ 317$ Abs. 3 BGB, also auch unterhalb der Schwelle qualifizierter Konzernierung z. B. Koppensteiner, in: Rowedder/ Schmidt-Leithoff, GmbHG, 4. Aufl., 2002, Anh. \$52 Rdn. 75; Altmeppen, Die Haftung des Managers im Konzern, 1998, S. 79 ff. 
Pflichtverletzung zurückzuführenden Schaden voraussetzt, woran es im qualifizierten faktischen Konzern gerade fehlte..$^{29}$

\section{Verlustausgleichspflicht als Folge einer Durchgriffshaftung bei missbräuchlichem Verbalten}

Nachdem bereits die „klarstellende“ „TBB“-Entscheidung ${ }^{30}$ in Wahrheit die Abkehr von dem Modell einer konzerngebundenen Haftung, hin zu einer allgemeinen Verhaltenshaftung darstellte, ${ }^{31}$ zog der BGH schließlich im Jahr 2001 mit der Entscheidung „Bremer Vulkan“32 die dogmatischen Konsequenzen aus seiner Kehrtwende und beerdigte die Statushaftung im qualifizierten faktischen Konzern, als er feststellte: „Der Schutz einer abhängigen $\mathrm{GmbH}$ gegen Eingriffe ihres Alleingesellschafters folgt nicht dem Haftungssystem des Konzernrechts des Aktienrechts ( $\mathbb{S} 291 \mathrm{ff}, 311 \mathrm{ff} A k t G)$ [...]." Die durch das reine Negativtestat offen gelassene Frage nach der dogmatischen Grundlage der im Verhältnis zu den Kapitalerhaltungsvorschriften aus $\$ \$ 30$, $31 \mathrm{GmbHG}$ subsidiären Haftung beantwortete der BGH wenig später im „KBV“-Urteil. ${ }^{33}$ Die Gesellschafter gingen in den Fällen der Existenzvernichtung ihres Haftungsprivilegs nach $\$ 13$ Abs. 2 GmbHG verlustig, wodurch die Verantwortlichkeit der Gesellschafter als eine Durchgriffshaftung beschrieben wird. Unabhängig davon, ob es sich insoweit um eine unbeschränkte und persönliche Außenhaftung ${ }^{34}$ gegenüber den Gläubigern oder doch um eine vom Insolvenzverwalter geltend zu machende Binnenhaftung ${ }^{35}$ handelte, war doch dogmatisch präjudiziert, dass Haftungsadressat notwendig nur der nach Wegfall des Haftungsschilds exponierte Gesellschafter sein konnte. ${ }^{36}$

$29 \mathrm{Zu}$ diesem Aspekt eingehend MüLBERT, Aktiengesellschaft, Unternehmensgruppe und Kapitalmarkt, 2. Aufl., 1996, S. 487 ff; zusammenfassend GoETte, Die GmbH, 2. Aufl., 2002, $\mathbb{\int} 9$ Rdn. 32 .

30 BGHZ 122, 123.

31 Hommelhoff, ZGR 1994, 395, 400 f; KrIEger, in: Münchener Handbuch des Gesellschaftsrechts, Band 4, 2. Aufl., 1999, $\$ 69$ Rdn. 115; LutTER, JZ 1993, 580 f; vgl. auch Goette, aaO (Fn. 29), $\$ 9$ Rdn. $19 \mathrm{ff}$.

32 BGHZ 149, 10 ff; zust. Altmeppen, NJW 2002, 321 ff; K. Schmidt, NJW 2001, 3577 ff; Ulmer, ZIP 2001, $2021 \mathrm{ff}$; Westermann, NZG 2002, $1129 \mathrm{ff.}$

33 BGHZ 151, 181 ff; Ulmer, JZ 2002, 1049 ff; Westermann, NZG 2002, 1129 ff; WilHELM, NJW 2003, $175 \mathrm{ff}$.

34 Lutter/Hommelhoff, GmbHG, 16. Aufl., 2004; $\$ 13$ Rdn. 24.

35 Altmeppen, NJW 2002, 321 ff, Ders., ZIP 2002, 1553 ff; Ders., ZIP 2001, 1837 ff; K. Schmidt, NJW 2001, $3577 \mathrm{ff}$; Wilhelm, NJW 2003, $175 \mathrm{ff}$.

36 Der Rückgriff auf $₫ 309$ Abs. 2 AktG schied konsequenterweise aus, BGHZ 149, 10, $16 \mathrm{f}$. Vgl. aber auch BGHZ 151, 181, $183 \mathrm{ff}$ „KBV“ und darauf rekurrierend PAEFGEN, in: Ulmer/Habersack/Winter, GmbHG, Bd. 2, 2006, \43 Rdn. 223; DERs., DB 2007, 1907, 1911, der bereits vor „Trihotel“ neben der Existenzvernichtungshaftung der Gesellschafter einen gegen die Geschäftsführer gerichteten Anspruch aus $\ 826$ BGB bei 
Letztere Konsequenz trifft auch für diejenigen Versuche in der Literatur zu, die Existenzvernichtungshaftung aus einer (gläubigerbezogenen) gesellschafterlichen Treuepflicht herzuleiten. ${ }^{37}$ Auch insoweit suggeriert die Anknüpfung an die Gesellschafterstellung eine zwangsläufige Beschränkung auf bestimmungsmächtige Verbandsmitglieder. Immerhin begründet aber das Abstellen auf fiduziarische Bindungen eine gewisse Offenheit des Haftungskonzepts, da die aus diesen entspringenden Verhaltenspflichten nicht prinzipiell auf Gesellschafter beschränkt sein müssen. Ganz Ähnliches gilt für die Versuche, die Verantwortlichkeit auf eine quasi-Organstellung des Alleingesellschafters im Verhältnis zur abhängigen Tochter zu gründen. ${ }^{38} \mathrm{Zwar}$ knüpft auch diese Konzeption an die beteiligungsbasierte Leitungsmacht des herrschenden Gesellschafters an, muss aber eine Erstreckung der Verschuldenshaftung der Quasi-Organe auf die Mitglieder der Verwaltung des herrschenden Gesellschafters noch nicht aus prinzipiellen dogmatischen Erwägungen ablehnen.

\section{Deliktische Verhaltenshaftung der Bestandsverantwortlichen}

Seit der Entscheidung „Trihotel“ ${ }^{\text {39 }}$ ordnet der 2. Zivilsenat die missbräuchliche Beeinträchtigung des Gesellschaftsvermögens nunmehr als sittenwidrige Schädigung ein und verortet ihre Sanktion folglich in der deliktischen Generalklausel des $₫ 826$ BGB. Neben der Aufgabe der Durchgriffs- zugunsten einer Schadensersatzhaftung ${ }^{40}$ kehrt der BGH von der seit „Bremer Vulkan“ geltenden Außenhaftung zum Modell einer Binnenverantwortlichkeit gegenüber der Gesellschaft zurück. Darüber hinaus wird die uneingeschränkte Ergebnis- durch eine Verschuldenshaftung ${ }^{41}$ abgelöst, die zudem in Idealkonkurrenz zu den Kapitalerhaltungsvorschriften der $\$ \mathbb{S} 30 \mathrm{f}$ GmbHG steht. Im Gegensatz zu den vormaligen objektiven Missbrauchstatbeständen ist Grund-

„weitgehender Deckungsgleichheit der Anspruchsvoraussetzungen“ bejaht. Ebenfalls vor Trihotel behandelt Wagner, FS Claus-Wilhelm Canaris, Bd. II, S. 473, $489 \mathrm{ff}$ die Existenzvernichtungshaftung als Anwendungsfall des $\ 826$ BGB mit einer Haftung auch der Organwalter.

37 Z. B. Scholz/Emmerich, Komm. z. GmbHG, Bd. 1, 10. Aufl., 2006; $\mathbb{1 3}$ Rdn. 92 ff; K. SChMidt, NJW 2001, 3577, 3580.

38 Insbesondere Altmeppen, in: Altmeppen/Roth, GmbHG, 5. Aufl., 2005, $\$ 13$ Rdn. 80; DERs., ZHR 171 (2007), 320, 329 ff; DERs., FS Priester, 2007, S. 1, 2 ff; zuletzt nochmals eingehend zum Haftungskonzept Ders., Münchener Komm. z. AktG, Bd. 5, 3. Aufl., 2010, $₫ 309$ Rdn. 2, $₫ 311$ Rdn. $316 \mathrm{ff}, \$ 317$ Rdn. $8 \mathrm{ff}$.

39 BGHZ 173, 246 ff; hierzu Altmeppen, NJW 2007, 2657 ff; Habersack, ZGR 2008, 533 ff; Osterloh-Konrad, ZHR 172 (2008), 274 ff; krit. Liebscher, aaO (Fn. 5), $\$ 13$ GmbHG Anh. Rdn. 534 .

40 Kleindiek, NZG 2008, 686, 687.

41 Altmeppen, NJW 2007, 2657; Osterloh-Konrad, ZHR 172 (2008), 274, 290. 
lage des $₫ 826$ BGB nun die Anknüpfung an eine subjektive Pflicht, deren schuldhafte Verletzung den Haftungstatbestand auslöst. ${ }^{42}$

Für die Verantwortlichkeit der Leitungsorgane im Konzern bedeutet die Abkehr von notwendig mitgliedschaftsbasierten Haftungsfundamenten des Gesellschaftsrechts und die Hinwendung zu allgemein bürgerlich-rechtlichen Instituten, dass der einschlägige Tatbestand der vorsätzlichen, sittenwidrigen Schädigung prinzipiell auch durch sie verwirklicht werden kann. Die in diesem Sinne im Grundsatz offene Passivlegitimation darf indes nicht darüber hinwegtäuschen, dass die Schädigungspotentiale, denen mit der Existenzvernichtungshaftung begegnet werden soll, originär im Organisationsrecht wurzeln. Die entscheidende Gläubigerbenachteiligung resultiert aus der Trennung von Vermögenssphären, die durch das Organisationsrecht erst ermöglicht wird; ${ }^{43}$ sie kann daher nur im verbandsrechtlichen Kontext begegnen. Die im Rahmen des $\$ 826 \mathrm{BGB}$ verletzte Pflicht ist also keinesfalls eine „Jedermann-Pflicht" ${ }^{44}$ - was nicht zuletzt in der bereits erwähnten Beschreibung der auf $\ 826$ BGB gestützten Existenzvernichtungshaftung als Sonderdeliktshaftung zum Ausdruck kommt. ${ }^{45}$ Zugespitzt formuliert, lässt sich der Auffassung des Bundesgerichtshofs, wonach es neben der Haftung aus $\ 826$ BGB „eines ... gesellschaftsrechtlich fundierten Haftungsinstituts" nicht bedürfe, ${ }^{46}$ allenfalls mit der Maßgabe zustimmen, dass die Existenzvernichtungshaftung ohnehin ein solches darstellt und daher die verbandsrechtlichen Wertungen bereits inkorporiert. ${ }^{47}$

Mit dem Vorgesagten ist freilich noch nicht ausgeschlossen, insoweit auch jenseits mitgliedschaftlicher Beteiligungen ${ }^{48}$ Akteure in die Pflicht zu nehmen, die bei der Ausübung verbandsrechtlicher Leitungsmacht über entsprechendes Schädigungspotential verfügen. Im Hinblick auf die gesetzlichen Vertreter

42 Altmeppen, NJW 2007, 2657, 2658.

43 Eingehend hierzu Tröger, FS Harm Peter Westermann, 2008, S. 1533, $1547 \mathrm{ff}$.

44 Osterloh-Konrad, ZHR 172 (2008), 274, 292.

45 Vgl. oben Fn. 8.

46 BGHZ 173, 246 Rdn. 29.

47 Das Verdikt des BGH ist v. a. gegen Versuche gerichtet, neben der Vorsatz erfordernden Sonderdeliktshaftung einen strengeren Haftungstatbestand auf der Grundlage einer schuldhaften Verletzung von Treupflichten zu etablieren, vgl. neben den in Fn. 35 Genannten nach der Trihotel-Entscheidung v.a. HüFrer, AktG, 9. Aufl., 2010, $\mathbb{1}$ Rdn. 26a. Insoweit ist darauf hinzuweisen, dass BGHZ 129, 136, $162 \mathrm{ff}$ "Girmes“ jedenfalls für schadenstiftende Stimmrechtsausübungen in der Hauptversammlung ebenfalls von einer Haftung nur für Vorsatz ausgeht. Verallgemeinernd BungEROTH, Münchener Komm. z. AktG, Bd. 1, 3. Aufl., 2008, \53a Rdn. 44; für diligentia quam in suis z. B. Hüffer, aaO, \53a AktG Rdn. 21. Gegen Treupflichtlösungen im Kontext der klassischen Existenzvernichtungstatbestände ZöLlNER, FS Konzern, 2006, S. 999, 1011.

48 Bereits in der Leitentscheidung bejaht der BGH die Haftung des nur mittelbar beteiligten Gesellschafters, BGHZ 173, 246 Rdn. 44. 
der Obergesellschaft gilt es daher, sich auf die normativen Fundamente ihrer Bindung zu besinnen und diese konzerndimensional zu entwickeln.

\section{Legitimation der Verantwortlichkeit auf der Grundlage bestehender Prinzipal-Agenten-Beziebungen}

Die analytische Kraft der Agenturtheorie zur Beschreibung verbandsrechtlicher Konfliktlagen ist im kapitalgesellschaftsrechtlichen Schrifttum in vielfältiger Weise fruchtbar gemacht worden. ${ }^{49} \mathrm{Im}$ Zentrum der bahnbrechenden, institutionenökonomischen Untersuchungen standen die Verhaltensanreize des Managements im Verhältnis zu den Anteilseignern in Publikumsgesellschaften. ${ }^{50}$ Die gewonnen Einsichten werden auch in der deutschsprachigen Literatur herangezogen, um die normativen Grundlagen der fiduziarischen Bindung der Leitungsorgane an die verbandsbezogenen Interessen der Anteilseigner zu beleuchten. Die Sanktion des Verstoßes gegen Zweck- und Treubindung über eine Schadensersatz-Verantwortlichkeit der Organwalter gegenüber der Gesellschaft ${ }^{51}$ nach $\$ \$ 93,116 \mathrm{AktG}, \mathbb{} \$ 3 \mathrm{GmbHG}$ wird als ein Weg analysiert, die kollektiven Interessen der Anteilseigner (auch) durch haftungsinduzierte Anreize abzusichern. ${ }^{52}$

Für die hier untersuchte Fragestellung ist entscheidend, dass nicht nur der vertikale Prinzipal-Agenten-Konflikt zwischen zersplitterten Anteilseignern und dem Management mit Hilfe des analytischen Instrumentariums der Agenturtheorie erhellt werden kann. Die grundlegende Einsicht, dass Wissenslücken von Prinzipalen den zur Verfolgung fremder Interessen eingesetzten Agenten die Gelegenheit bieten, ihre eigenen Ziele auf opportunistische Weise

49 Allgemein zum Erkenntnispotential der Agenturtheorie im Verbandsrecht, FLEISCHER, ZGR 2001, 1, 7 ff Umfassende, funktional-rechtsvergleichende Darstellung fundamentaler Institute des Kapitalgesellschaftsrechts auf der Grundlage der Agenturtheorie in Kraakman/Armour/Davies/Enriques/Hansmann/Hertig/Hopt/Kanda/Rock, The Anatomy of Corporate Law, 2. Aufl., 2009.

50 Jensen/Meckling, 3 J. Fin. Econ. 305, 308 (1976); zuvor auch schon Alchian/DemSETZ, 72 Am. Econ. Rev. 777, 779 ff (1972). Vgl. auch BARZEL, 25 J.L. \& Econ. 27 (1982); FAma/Jensen, 26 J.L. \& Econ. 301 (1983); DiEs., 26 J.L. \& Econ. 327 (1983). Überblick zum gegenwärtigen Stand der Agenturtheorie in der Institutionenökonomik bei ERLEI/ LeschKe/SauerLand, Neue Institutionenökonomik, 2. Aufl., 2007, S. $69 \mathrm{ff}$.

51 Die Aktivlegitimation der Gesellschaft dient im Ausgangspunkt ganz ähnlichen Zielen wie eine class action, weil auch sie darauf abzielt, die Interessen einer geschädigten Gruppe im Haftungsprozess zu vereinigen. Zur class action im verbandsrechtlichen Kontext, die im U.S.-amerikanischen Schrifttum durchaus als Substitut des derivative suit gesehen wird, Romano, 7 J.L. Econ. \& Org. 55 (1991); KraAkman/Parks/Shavell, Geo. L. Rev. 1733 (1994).

52 Monographische Analyse auf der Grundlage der Prinzipal-Agenten-Theorie bei ARNOLD, Die Steuerung des Vorstandshandelns, 2007. 
anstelle derjenigen der Prinzipale zu verfolgen, ist vielmehr auch geeignet, eine horizontale Prinzipal-Agenten-Beziehung zu beschreiben, ${ }^{53}$ wie sie vor allem in konzernrechtlichen Abhängigkeitslagen zwischen Mehrheits- und Minderheitsaktionären in Form des paradigmatischen „Konzernkonflikts“ begegnet. ${ }^{54}$ Schließlich ermöglicht der Ansatz, die Verhaltensanreize im Verhältnis von Eigen- zu Fremdkapitalgebern mit samt den institutionellen Steuerungsmechanismen zu beschreiben, die potentiellem Opportunismus - insbesondere von bestimmungsmächtigen Gesellschaftern - entgegenwirken. ${ }^{55}$ Grob gesprochen stellt die Haftungssanktion einen rechtsförmigen Steuerungsmechanismus dar, der zur Internalisierung solcher Handlungsfolgen der Agenten zwingt, die andernfalls den Prinzipalen zur Last fielen. ${ }^{56} \mathrm{Im}$ konkreten Kontext geht es darum, die sozial nicht wünschenswerte Ausbeutung von Minderheitsaktionären und Gläubigern zu verhindern und so die mit der spezialisierten Rollenverteilung einhergehenden Vertretungskosten (agency cost) $\mathrm{zu}$ minimieren. Unter diesem anreizorientierten Blickwinkel ${ }^{57}$ kommt eine Ausdehnung der Existenzvernichtungshaftung auf Organwalter dann in Betracht, wenn die Haftung nur des bestimmungsmächtigen Gesellschafters zu Steuerungsdefiziten führt, z. B. weil bindende Vermögensrestriktionen seine Verantwortlichkeit teilweise leer laufen lassen (judgement proof) $)^{58}$ und sonstige Vollzugsdefizite, die unerwünschte (partielle) Externalisierung der Handlungsfolgen erlauben.

Das Wegreißen des Haftungsschilds des $₫ 13$ Abs. 2 GmbHG als Folge der Sonderdeliktshaftung führt bei der Ein-Mann Gesellschaft bzw. bei einvernehmlich zusammenwirkenden Geschäftsleiter-Gesellschaftern zu einer zutreffenden, die Gläubigerinteressen prinzipiell ${ }^{59}$ adäquat berücksichtigenden

53 Vgl. Roe, in: Ménard/Shirley (Hrsg.), Handbook of New Institutional Economics, 2008, S. 371, $374 \mathrm{f}$.

54 Beschreibung des „Konzernkonflikts“ im deutschsprachigen juristischen Schrifttum z. B. bei KropfF, Aktiengesetz, 1965, S. 273 f; Koppensteiner, Kölner Komm. z. AktG, 3. Aufl., Bd.6, 2004, Vor $\$ 291$ Rdn. 5 ff; Altmeppen, aaO (Fn. 38), Vor $\$ 311$ AktG Rdn. 1 f; Habersack, aaO (Fn. 8), $\$ 311$ Rdn. 1.

55 Grundlegend insoweit Myers, 5 J. Fin. Econ. 147 (1977) ; aber auch schon Jensen/ Meckling, 3 J. Fin. Econ. 305, 334 ff (1976).

56 Überblick über die formale Prinzipal-Agenten-Theorie bei ErLei/LeschKe/SAuerLAND, aaO (Fn. 50), S. 109 ff; vgl. auch allgemein Richter/Furobotn, Neue Institutionenökonomik, 4. Aufl., 2010, S. $173 \mathrm{ff}, 224 \mathrm{ff}$.

57 Insofern ist zu betonen, dass auch der BGH die verhaltenssteuernde Zielsetzung der von ihm etablierten Existenzvernichtungshaftung ausdrücklich anerkennt, wenn er von einem Schutz der Gesellschaftsgläubiger „durch die repressive Anordnung der Schadensersatzpflicht“ spricht, BGHZ 176, 204 Rdn. 13 „Gamma“.

58 Speziell hierzu Shavell, 6 Int'l Rev. L \& Econ. 45 (1986).

59 Auch insofern bestehen Grenzen aus einer bindenden Budgetrestriktion, die freilich insoweit kein originär, durch die Haftungsbeschränkung des Verbandsrechts erzeugtes Problem darstellt. 
Motivationslage aller Entscheidungsträger. Ersichtlich besteht allenfalls im Fall der Fremdorganschaft ein Bedürfnis nach einer parallelen Verantwortlichkeit der Organwalter, um auch insoweit eine Internalisierung der gläubigerbetreffenden Auswirkungen der Geschäftsführung zu erzwingen. Ein solches Bedürfnis kann, unabhängig von Verbundlagen deshalb bestehen, weil und soweit die Organwalter im operativen Geschäft wegen der Distanz der Gesellschafter de facto bestimmungsmächtig sind. Es kann aber auch daraus resultieren, dass die prinzipiell an einer kurzen Leine gehaltenen Geschäftsleiter durch die Sanktionsdrohung angehalten werden sollen, Anreizen der Eigenkapitalgeber entgegenzusteuern, die Gläubiger zu benachteiligen, wie sie sich gerade in Insolvenznähe aktualisieren. ${ }^{60}$ Die Geschäftsführer können, trotz der prinzipiellen Weisungsbefugnis des höchsten Organs der $\mathrm{GmbH}$, existenzgefährdende Weisungen als nichtig unbeachtet lassen ${ }^{61}$ - wozu sie durch eine an sie gerichtete, scharfe Haftungssanktion im Gläubigerinteresse angehalten werden können. Im Unternehmensverbund tritt hinzu, dass die Internalisierungswirkung der Gesellschafterhaftung beschränkt ist, wenn das herrschende Unternehmen seinerseits eine nur mit dem eingelegten Vermögen haftende Einheit darstellt (vgl. z. B. $\mathbb{\int} 13$ Abs. 2 GmbHG, $\mathbb{} 1$ Abs. 1 Satz 2 AktG). Die Haftung des herrschenden Gesellschafters nach $\$ 826$ BGB erreicht dann das Privatvermögen seiner Residualgläubiger nicht. In der Konsequenz besteht auch in diesen Konstellationen das Bedürfnis, über eine eigene Verantwortlichkeit der Geschäftsleiter der Obergesellschaft deren Eigeninteresse zu aktivieren, ein Gegengewicht zu den Anreizen der Anteilseigner zu bilden, hinter dem Haftungsschild in der Krise zu Hasardeurritten anzusetzen.

Das Vorgesagte legitimiert allerdings in den beschriebenen Konstellationen nur allgemein eine rechtsförmig abgesicherte Mitverantwortlichkeit der Organe des bestimmungsmächtigen Gesellschafters im Hinblick auf die Interessen der Gläubiger (der Untergesellschaft). Im Rahmen eines Institutionenvergleichs präjudizieren die angestellten Überlegungen also weder die Gestalt, noch gar die dogmatische Umsetzung der wünschenswerten Pflichtenbindung. Einer Erstreckung gerade der Sonderdeliktshaftung wegen Existenzvernichtung bedürfte es vor diesem Hintergrund nur dann, wenn die auf eine entsprechende Mitverantwortung abzielenden Institute des Verbandsrechts nicht hinnehmbare Insuffizienzen aufwiesen. Somit sind im Folgenden die jenseits des $₫ 826$ BGB bestehenden Pflichtenbindungen der Fremdorgane

60 Eine drohende Eigenhaftung der Gesellschafter entfaltet dann keine Anreizwirkung, wenn deren wirtschaftliche Existenz mit derjenigen der Gesellschaft so eng verwoben ist, dass die Insolvenz des Verbands auch privat materiell vernichtende Wirkungen zeitigt. Zum Ganzen auch KLÖHN, ZGR 2008, 110, 112 ff.

61 Vgl. nur Altmeppen, aaO (Fn.5), \37 GmbHG Rdn.7; Scholz/U. Schneider, Komm. z. GmbHG, Bd.2, 10 Aufl., 2007, 337 Rdn. 52; KleindieK, in: Lutter/Hommelhoff, GmbHG, 17. Aufl., 2009, \37 Rdn. 18; Wicke, GmbHG, 2008, 337 Rdn. 5. 
samt ihrer Durchsetzungsmechanismen in den Blick zu nehmen und auf ihre Leistungsfähigkeit in der Verbundlage zu untersuchen.

\section{Konzernspezifische, fiduziarische Bindungen der Leitungsorgane im deutschen Gesellschaftsrecht}

\section{Leitungskompetenz und-verantwortung im Konzern}

\section{a) Konzernspezifische Organpflichten gemäß $\int \mathbb{S} 76,93$ Akt $G$ und $\int 43 G m b H G$}

Ausgangspunkt der Überlegungen ist die unstreitige Sicht, dass zu den zu beobachtenden Pflichten eines ordentlichen und gewissenhaften Geschäftsleiters der herrschenden Gesellschaft jedenfalls gehört, die aus den gehaltenen Beteiligungen erwachsenden Rechte in einer Art und Weise wahrzunehmen, die der Erreichung der Verbandsziele dient. ${ }^{62}$ Umstritten ist freilich, ob und gegebenenfalls mit welcher Intensität der Vorstand der Obergesellschaft, insbesondere im faktischen Konzern, ${ }^{63}$ verbundintegrative Leitungsmacht ausüben muss, um dieser konzerndimensionalen Verantwortung zu genügen. Die Kontroverse zwischen den vereinzelten Befürwortern einer intensiven Konzernintegrationspflicht ${ }^{64}$ und den ganz überwiegenden Kritikern dieser Posi-

62 Für den Vorstand z. B. Mertens/Cahn, Kölner Komm. z. AktG, Bd. 2/1, 3. Aufl., 2010, \76 Rdn. 65; Koppensteiner, aaO (Fn. 54), Vorbem. \291 AktG Rdn. 71; Spindler, Münchener Komm. z. AktG, Bd. 2, 3. Aufl., 2008, \$76 Rdn. 49; KorT, Großkomm. z. AktG, 4. Aufl., 2002, $\$ 76$ Rdn. 140; Норт, Großkomm. z. AktG, 4. Aufl., 1999, $\$ 93$ Rdn. 114; Hüffer, aaO (Fn. 47), $\$ 76$ AktG Rdn.17a; Fleischer, in: Spindler/Stilz, AktG, Bd. 1, 2. Aufl., 2010, $\$ 76$ Rdn. 91; RAISER/VeIL, Recht der Kapitalgesellschaften, 5. Aufl., 2010, \$14 Rdn.90; Timm, Die Aktiengesellschaft als Konzernspitze, 1980, S. 95 ff. Für den Geschäftsführer z. B. Jungkurth, Konzernleitung bei der $\mathrm{GmbH}$, 2000, S. 57 ff; Kleindiek, aaO (Fn. 61), \43 GmbHG Rdn. 11; Altmeppen, aaO (Fn. 5), $\$ 43$ GmbHG Rdn. 12; Scholz/U. Schneider, aaO (Fn. 61), \43 GmbHG Rdn. $47 \mathrm{ff}$.

63 Für den Vorstand im Vertragskonzern bejahend im Sinne einer intensiven Pflicht zur integrativen Verbundleitung z.B Hommelhoff, Die Konzernleitungspflicht, 1982, S. 43 ff; Bayer, Münchener Komm. z. AktG, Bd.1, 3. Aufl., 2008, \$18 Rdn. 19; KrieGER, aaO (Fn. 31), \$70 Rdn. 155; abl. Mertens/Cahn, aaO (Fn. 62), \$76 AktG Rdn. 65; Spindler, aaO (Fn. 62), \$76 AktG Rdn. 49; Kort, aaO (Fn. 62), \$76 AktG Rdn. 144; Hüffer, aaO (Fn. 47), $\$ 76$ AktG Rdn.17a; Fleischer, aaO (Fn.62), \$76 AktG Rdn. 90; Löвве, Unternehmenskontrolle im Konzern, 2003, S. 83; GöTZ, ZGR 1998, 524, 526 ff. Zur GmbH Jungkurth, aaO (Fn. 62), S. 57 ff; Kleindiek, aaO (Fn. 61), §37 GmbHG Rdn. 6.

64 Grundlegend auch insoweit Hommelhoff, aaO (Fn. 63), S. 165 ff, 184 ff; zust. Kropf, ZGR 1984, 112, 116. Vgl. auch U. SchneIder/S. SChNeIDER, ZIP 2007, 2061, 2062, die zumindest eine Leitungspflicht des Vorstands der Obergesellschaft, wenn auch ohne Konzernfinalität annehmen. 
tion, ${ }^{65}$ muss für die hier interessierende Fragestellung jedoch nicht entschieden werden: Unabhängig von der genauen Reichweite des Geschäftsleiterermessens gehört es nämlich zu den allseits geteilten Ankerpunkten der Debatte, dass jedenfalls solche Leitungsmaßnahmen mit der Zweckförderungspflicht des Vorstands unvereinbar sind, die zu einer Schädigung der Tochter führen, die auch die Obergesellschaft benachteiligt, weil sie vermittelt über weitreichende Haftungstatbestände auf diese zurückschlägt. ${ }^{66}$ Dementsprechend kann das Auslösen einer Verantwortlichkeit der Konzernmutter wegen Existenzvernichtung der Untergesellschaft nicht mehr zu den Maßnahmen gehören, die im Leitungsermessen des jeweiligen Organs stehen. Insoweit erscheint es auch nicht entscheidend, dass die verbandsrechtlichen Grenzen der Einflussnahme aus dem Organisationsrecht der Untergesellschaft, wie insbesondere die $\int \mathbb{S} 311 \mathrm{ff} A k t G$ oder das treupflichtbasierte Schädigungsverbot des GmbH-67 bzw. Personengesellschaftsrechts, ${ }^{68}$ an sich unmittelbar an die Obergesellschaft als herrschendes Unternehmen bzw. Mitglied des Verbands gerichtet sind. Auch eine nur reflexartige Relevanz für die Pflichten der Organwalter führt im hier untersuchten Kontext zu deren Haftung im Falle der Missachtung.

\section{b) Binnenhaftung auf der Ebene der Obergesellschaft als (unzureichende) Sanktion}

Verletzt der Geschäftsleiter die Pflicht einer verantwortungsvollen Verbundleitung, so löst dies nach Maßgabe der $\mathbb{S} 93$ Abs. 2 Satz 1 AktG, $\mathbb{} \$ 3$ Abs. 2 GmbHG eine Schadensersatzpflicht aus. Diese bleibt jedoch aus Sicht der von der nachteiligen Geschäftsführungsmaßnahme betroffenen Untergesellschaft und ihrer Gläubiger aus mehreren Gründen ein stumpfes Schwert. Zum einen handelt es sich bei der Haftung wegen pflicht- oder sorgfaltswidriger

65 In diesem Sinne HüFfER, aaO (Fn. 47), $\$ 76$ AktG Rdn. 17; DERs., in: Bayer/Habersack (Hrsg.), Aktienrecht im Wandel, 2007, \$7 Rdn. 24; SеIвт, in: K. Schmidt/Lutter (Hrsg.), AktG, Bd.1, 2. Aufl., 2010, $\$ 76$ Rdn.16; eingehend auch Mülbert, aaO (Fn.29), S. $29 \mathrm{ff}$.

66 Vgl. nur Hüffer, aaO (Fn. 47), \93 AktG Rdn. 15; U. Schneider, FS Uwe Hüffer, 2010, S. $905,910 \mathrm{ff}$.

67 BGHZ 65, 15, 19 f „ITT“; vgl. hierzu Altmeppen, aaO (Fn. 5), \$13 GmbHG Rdn. 37, Anh. zu $₫ 13$ GmbHG Rdn. 154 ff; Scholz/Emmerich, aaO (Fn. 37), Anh. zu $\$ 13$ GmbHG Rdn. 70; Habersack, aaO (Fn. 8), Anh. zu $\$ 318$ Rdn. 27 ff; Hueck/Fastrich, in: Baumbach/Hueck, GmbHG, 19. Aufl., 2010, $\$ 13$ Rdn. 20, 54; LutTer, aaO (Fn. 5), Anh. zu $₫ 13$ GmbHG Rdn. 21; RaIser, in: Ulmer/Habersack/Winter, GmbHG, 2005, $₫ 14 \mathrm{Rdn} .67 \mathrm{ff}$.

68 Vgl. nur Tröger in: Westermann, Handbuch der Personengesellschaften, Rdn. I 4051 (Loseblatt Stand 5/2008); Schäfer, Großkomm. z. HGB, Bd.3, 5. Aufl., 2009, Anh. \105 Rdn. 44; MüLbert, aaO (Fn. 13), KonzernR Rdn. 171 f. 
Geschäftsführung um eine Binnenhaftung, die lediglich einen Anspruch der „eigenen“ Gesellschaft, also der Obergesellschaft, begründet. Denn nur dieser gegenüber obliegen den Konzernvorständen und -geschäftsführern die oben aufgeführten Pflichten. ${ }^{69}$ Zum anderen ist eine Geltendmachung eventuell bestehender Ansprüche gegen die Organwalter der anstellenden Körperschaft nicht immer zu erwarten, sodass ihre Anreizwirkung eingeschränkt ist. Dies gilt insbesondere dann, wenn die zur Geltendmachung berufenen Organe in die haftungsauslösenden Vorgänge involviert waren. Entscheidend sind insoweit die Voraussetzungen für die Geltendmachung der Organhaftung im Organisationsrecht der Obergesellschaft, die im Aktienrecht trotz der UMAGReformen nach wie vor als Ursache eines Vollzugsdefizits der im Ausgangspunkt durchaus scharfen Verantwortlichkeit nach $\$ 93 \mathrm{AktG}$ angesehen werden können. ${ }^{70}$ Aus dem - den Kreis der Aktivlegitimierten erweiternden ${ }^{71}$ - Verfolgungsrecht des $\ 93$ Abs. 5 Satz 1 AktG, dessen entsprechende Anwendung für die Haftung nach $₫ 43$ Abs. $2 \mathrm{GmbHG}$ verbreitet befürwortet wird, ${ }^{72}$ können die geschädigten Gläubiger der Konzerntochter immerhin ei-

69 Für die AG Habersack, aaO (Fn. 8), \311 Rdn.11; Krieger, aaO (Fn.31), \69 Rdn. 21; Mertens/Cahn, aaO (Fn. 62), \$76 AktG Rdn.65; Spindler, aaO (Fn. 62), \$76 AktG Rdn.49ff; U. Schneider/S. Schneider, ZIP 2007, 2061, 2062; für die GmbH Jungkurth, aaO (Fn.62), S.94f; Kleindiek, aaO (Fn.61), \43 GmbHG Rdn. 11; Scholz/U. Schneider, aaO (Fn. 61), $\$ 43$ GmbHG Rdn. 47 ff.

70 Vgl. z. B. Peltzer, FS Uwe H. Schneider, 2011, S. 953, 954 ff; Lutter, FS Uwe H. Schneider, 2011, S. 763, 764 ff; Hellwig FS Georg Maier-Reimer, 2010, S. 201, 213 f; Mertens/ Cahn, aaO (Fn. 62), $\$ 93$ AktG Rdn. 6; RAISER/VeIL, aaO (Fn. 62), \14 Rdn. 97; SPINDLER, aaO (Fn. 62), $\$ 93$ AktG Rdn. 2; a. A. Hopt, aaO (Fn. 62); $\$ 93$ AktG Rdn. 16 f; WIESNER, in: Münchener Handbuch des Gesellschaftsrechts, Band 4, 3. Aufl., 2007, $\$ 26$ Rdn. 2. Speziell zum Klageerzwingungsverfahren nach $\mathbb{\$} \$ 148 \mathrm{f}$ AktG LiNNERz, NZG 2004,307,310; WACLAWIK, DStR 2006, 2177, 2182; zur Sonderprüfung auf Verlangen einer Minderheit JäNIG, BB 2005, 949; SЕIBT, WM 2004, 2137, $2139 \mathrm{ff}$.

71 Die h. M. geht insoweit von einer Anspruchsvervielfältigung aus, MERTEns/CAHN, aaO (Fn. 62), \93 AktG Rdn. 180; Spindler, aaO (Fn. 62), \93 AktG Rdn. 234; Hopt, aaO (Fn. 62), \93 AktG Rdn. 397 ff; Fleischer, aaO (Fn. 62), \93 AktG Rdn. 294; KrieGer/Sailer-Coceani in: K. Schmidt/Lutter, AktG, Bd. 1, 2. Aufl., 2010, \$93 Rdn. 55; Hüffer, aaO (Fn. 47), $\$ 93$ AktG Rdn. 32; Wiesner, aaO (Fn. 70), $\$ 26$ Rdn. 25; ZemPelin, AcP 155 (1956) 209, 241. Nach a. A. begründet $\$ 93$ Abs. 5 Satz 1 AktG eine gesetzliche Prozessstandschaft, z. B. Hueck, in: Baumbach/Hueck, AktG, 13. Aufl., 1968, \93 Anm. 15, 17; Godin/Wilhelmi, AktG, Bd.1, 1971, $\$ 93$ Anm. 28; НaвSCHEID, FS Friedrich Weber, 1975, S. 197.

72 Eingehend Wilhelm, Rechtsform und Haftung bei der juristischen Person, 1981, S. 363 ff. Mit Unterschieden im Detail z.B. Kleindiek, aaO (Fn. 61), $\$ 43$ GmbHG Rdn. 42; K. Sснмidt, Gesellschaftsrecht, 4. Aufl., 2002, \$9 IV 5, S. 244 ff; DERs., ZGR 1996, 209, 224; UlMER, ZIP 2001, 2021, 2028; beschränkt auf entsprechend $\ 93$ Abs. 5 Satz 2, Satz 3 AktG nicht disponible Ansprüche Altmeppen, aaO (Fn. 5), $₫ 43$ GmbHG Rdn. 88; für analog $\mathbb{\$} 93$ Abs. 3 Satz 3 BGB unverzichtbare Ansprüche PAefgen, aaO (Fn. 36), $\$ 43$ GmbHG Rdn. 174; im Fall der sonst scheiternden Anspruchsdurchsetzung gegen den Geschäftsführer ZöllnER/NoAcK, aaO (Fn. 10), \43 GmbHG Rdn. 31; 
nen reflexartigen Nutzen ziehen. Insoweit ist zu bedenken, dass zwar alleine die Gläubiger der Obergesellschaft, nicht hingegen diejenigen der Tochter den Ersatzanspruch gegen das Leitungsorgan geltend zu machen ermächtigt werden. Allerdings zählt die Untergesellschaft mit dem gegen ihr beherrschendes Mitglied gerichteten Anspruch aus $₫ 826$ BGB wegen Existenzvernichtung zu den Gläubigern der Obergesellschaft. Selbstredend ist nicht damit zu rechnen, dass die Geschäftsführer der werbenden Gesellschaft ohne Weiteres gegen die Organe ihrer Konzernmutter vorgehen. Keine entsprechende Zurückhaltung wird aber der - im Fall der Existenzvernichtung naturgemäß involvierte Insolvenzverwalter der Untergesellschaft an den Tag legen, wenn dieser die Haftung der Obergesellschaft aus $\$ 826$ BGB durchsetzt. Der dogmatische

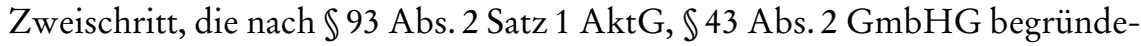
ten Organhaftungsansprüche der Obergesellschaft gemäß $₫ 93$ Abs. 5 Satz 1 AktG (analog) zu verfolgen, um anschließend vom herrschenden Unternehmen Befriedigung des Anspruchs aus $\ 826 \mathrm{BGB}$ zu erlangen, führt aus der Sicht der Geschäftsleiter der Mutter- prinzipiell zu einer vollen Internalisierung der Gläubigerschädigung auf der Ebene der Tochtergesellschaft. Dieser Befund gilt auch dann, wenn das individuelle Verfolgungsrecht der Gläubiger nach $\$ 93$ Abs. 5 Satz 4 BGB zugunsten des Insolvenzverwalters der Obergesellschaft suspendiert wird, da auch insoweit mit einem Vorgehen gegen die haftenden Verwaltungsmitglieder zu rechnen ist. Anreizdefizite drohen aber, wenn sich die herrschende Gesellschaft selbst nicht in Zahlungsschwierigkeiten befindet und daher weder das bedingte Verfolgungsrecht des Insolvenzverwalters der vernichteten Tochtergesellschaft nach oder entsprechend $\$ 93$ Abs. 5 Satz 1 AktG begründet ist, ${ }^{73}$ noch eine Zuständigkeit eines Insolvenzverwalters der Obergesellschaft nach $\$ 93$ Abs. 5 Satz 4 AktG eingreift. In dieser Konstellation besteht nur der - durch die ARAG-Judikatur verstärkte, ${ }^{74}$ in seiner praktischen Wirksamkeit gleichwohl nicht über jeden Zweifel erha-

ganz abl. Scholz/U. Schneider, aaO (Fn. 61), \43 GmbHG Rdn. 291; Mertens, in: Hachenburg, GmbHG, 8. Aufl., 1997, \43 Rdn. 99; ausführlich Sтовве, Die Durchsetzung gesellschaftsrechtlicher Ansprüche der $\mathrm{GmbH}$ in Insolvenz und masseloser Liquidation, 2001, S. $456 \mathrm{ff}$.

$73 \mathrm{Zu}$ den diesbezüglichen Voraussetzungen des Verfolgungsrechts nur Mertens/CaHn, aaO (Fn. 62), $\$ 93$ AktG Rdn. 182; SpIndler, aaO (Fn. 62), $\$ 93$ AktG Rdn. 238; Hopt, aaO (Fn. 62), $\$ 93$ AktG Rdn. 407; Fleischer, aaO (Fn. 62), 93 AktG Rdn. 300; KrIEger/Sailer-Coceani, aaO (Fn. 71), $₫ 93$ AktG Rdn. 60; Hüffer, aaO (Fn. 47), $\ 93$ AktG Rdn. 33.

74 Nach BGHZ 135, 244 „ARAG/Garmenbeck“ darf der Aufsichtsrat einer AG nur dann ausnahmsweise von der Verfolgung seiner Meinung nach bestehender Schadensersatzansprüche gegen den Vorstand absehen, wenn „gewichtige Gründe des Gesellschaftswohls dagegen sprechen und diese Umstände die Gründe, die für eine Rechtsverfolgung sprechen, überwiegen oder ihnen zumindest gleichwertig sind." Zusammenfassend Henze, NJW 1998, 3309. Dabei soll dem Aufsichtsrat bei seiner Entscheidung von Rechts wegen kein Ermessenspielraum zukommen, BGHZ 135, 244, 254; aus jüngerer Zeit Koch, WM 2009, 93; a. A. zuletzt wieder Paefgen, AG 2008, 761, 762 ff: Anwen- 
bene - Druck auf die Gesellschaft und die berufenen Organe, wegen der Klage des Insolvenzverwalters Regress bei den eigenen Verwaltungsmitgliedern zu nehmen. ${ }^{75}$

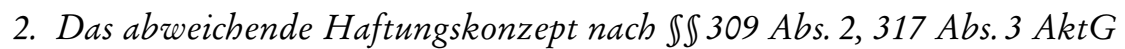
und die Parallelen im GmbH-Konzernrecht

Die Sonderbestimmungen des 3. Buches des AktG über die persönliche Verantwortlichkeit der Organwalter des herrschenden Unternehmens für die Verletzung verbundbezogener Pflichten ruhen auf einem normativen Fundament, das sich prinzipiell rechtsformunabhängig und auch dann als tragfähig erweist, wenn es um die Erstreckung der Sonderdeliktshaftung wegen Existenzvernichtung auf die Organwalter geht. Der Gedanke einer Einbeziehung auch der Verwaltungsmitglieder des herrschenden Unternehmens als Korrelat der verbundweiten Ausübung von Leitungsmacht ist nicht nur aus der Perspektive einer konzernfreien Minderheit, sondern auch vor dem Hintergrund des Schutzbedürfnisses der Gläubiger geboten.

\section{a) Haftungserstreckung als Korrelat konzerndimensionaler Leitungsmacht}

Nach $\int \mathbb{S} 309$ Abs. 2, 317 Abs. 3 AktG haften die gesetzlichen Vertreter der Obergesellschaft der Untergesellschaft unmittelbar für die Verletzung ihrer Konzernleitungspflichten, die in $\$ 309$ Abs. $1 \mathrm{AktG}$ explizit kodifiziert wurden bzw. als Folge der Gesamtschuldanordnung aus $\$ 317$ Abs. 1 AktG abzuleiten sind. Dies bedeutet im Vertragskonzern, dass sie für die Schäden verantwortlich sind, die der abhängigen Aktiengesellschaft aus der Erteilung von Weisungen erwachsen, die mit den Verhaltensstandards des $\$ 309$ Abs. $1 \mathrm{AktG}$ unvereinbar sind. Ganz ähnlich sind sie im faktischen Konzern der abhängigen Aktiengesellschaft gegenüber persönlich haftbar, wenn von ihnen Maßnahmen veranlasst wurden, die mit dem System des gestreckten Einzelausgleichs nach $\$ \llbracket 311 \mathrm{ff} A k t G$ unvereinbar sind. Es kann dahinstehen, ob die Haftungsbestimmungen als Ausdruck einer quasi-Organstellung der Verwaltungsmit-

dung der business judgement rule; zustimmend Mertens, FS Karsten Schmidt, 2009, S. 1183, 1195 mit kritischer Analyse der Judikatur, ibid. S. $1186 \mathrm{ff}$.

75 Die bekannten Durchsetzungsdefizite insbes. der aktienrechtlichen Binnenhaftung werden ausgeblendet bei, S. SchneIder, FS Uwe H. Schneider, S. 1177, 1195. An deren prinzipieller Relevanz ändert auch der Umstand nichts, dass bei - keinesfalls zwingend bestehenden - Doppelmandaten, ein auch auf der Ebene der insolventen Tochter$\mathrm{GmbH}$ agierender Organwalter des beteiligten Verbands in manchen Fällen nach $₫ 823$

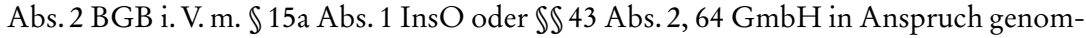
men werden kann: Der haftungsinduzierte Gläubigerschutz bleibt konzeptionell lückenhaft. 
glieder des herrschenden Unternehmens auf der Ebene der abhängigen Aktiengesellschaft interpretiert werden können. ${ }^{76}$ Entscheidend ist allein, dass sie dem Umstand Rechnung tragen, dass zwar die Leitungsmacht der gesetzlichen Vertreter im Konzern über den eigenen Verband hinausreicht, diesem Machtzuwachs aber mit der auf das Binnenverhältnis zur Anstellungskörperschaft beschränkten Erweiterung der haftungsbewährten Verhaltensstandards nicht adäquat Rechnung getragen werden kann. Es entspricht dem Regelungskonzept des Gesetzes, dem mitgliedschaftlich begründeten, im Rahmen der Beteiligungsverwaltung von den Leitungsorganen des herrschenden Unternehmens realisierbaren Schädigungspotential mit einer verbandsrechtlichen Haftungsandrohung entgegenzuwirken. ${ }^{77}$ Diese bietet - trotz vielfältiger, auf empfundene Defizite reagierenden Reformvorschläge ${ }^{78}$ - im konzeptionellen Ausgangspunkt erhöhte Gewähr für die Durchsetzung der als angemessen empfundenen Verhaltensstandards. Das legislatorische Bemühen, ein gerade der Konzernlage gerecht werdendes, Leitungsmacht und -verantwortlichkeit effektiv parallelisierendes Sanktionensystem zu schaffen, wird nicht zuletzt durch das Einzelklagerecht der Aktionäre aus $\$ \int 309$ Abs. 4 Satz 1, 317 Abs. 4 AktG verdeutlicht, das nicht nur die offensichtlich dysfunktionale Zuständigkeit des Vorstands der abhängigen Aktiengesellschaft überwindet, sondern auch eine spürbare Erleichterung gegenüber den im Konzern nur bei Annahme eines breiten Stimmverbots des herrschenden Unternehmens und seiner Affiliierten ${ }^{79}$ einigermaßen effektiv handhabbaren Anforderungen des $₫ 147$ AktG darstellt. ${ }^{80}$ Ganz ähnlich wird auch das bereits in $\$ 93$ Abs. 5 AktG begegnende Gläubigerverfolgungsrecht in $\int \mathbb{S} 309$ Abs. 4 Satz 3, 317 Abs. 4

76 In diese Richtung insbesondere die auf Marcus LutTer (FS Robert Fischer, 1979, S. 419; FS Walter Stimpel, 1985, S. 825, 827 ff; ZGR 1987, 324, 329 ff) zurückgehenden Versuche, die wirtschaftliche Einheit des Unternehmensverbunds auch rechtlich als solche zu verfassen, vgl. zu dieser Agenda U. H. Schneider, in: Mestmäcker/Behrens (Hrsg.), Das Gesellschaftsrecht der Konzerne im internationalen Vergleich, 1991, S. 563 ff. Dagegen z. B. BäLz, AG 1992, 277, 292; Mülbert, aaO (Fn. 29), S. 25; TröGER, Treupflicht im Konzernrecht, 2000, S. $1 \mathrm{ff}$.

77 Vgl. hierzu BegrRegE bei KropfF, Aktiengesetz, 1965, S. 404; Koppensteiner, aaO (Fn. 54), \309 AktG Rdn. 1; Hirte, Großkomm. z. AktG, 4. Aufl., 2005, \$309 Rdn. 3; Altmeppen, aaO (Fn. 38), $\$ 309$ AktG Rdn. 1 f; $\$ 317$ AktG Rdn. 87; Hüffer, aaO (Fn. 47), \309 AktG Rdn. 1; Emmerich, Gedächtnisschrift Jürgen Sonnenschein, 2003, S. $651,652 \mathrm{ff}$.

78 Vgl. die - von der wirtschaftsrechtlichen Abteilung des 59. DJT allerdings nicht aufgegriffenen - Anregungen bei Hommelhoff, Gutachten z. 59. DJT, 1992, S. G 7, 67.

79 Dazu Altmeppen, aaO (Fn. 38), $\$ 317$ AktG Rdn. 70, 104.

80 In der Lit. ist umstritten, ob die Ansprüche gegen das herrschende Unternehmen und seine Verwaltungsmitglieder aus $\mathbb{\$} \$ 309,317$ AktG dem Mechanismus des beschlussbedingten Klagezwangs unterliegen. Befürwortend OLG München, ZIP 2008, 1916, 1918 re. Sp.; KropfF, FS Bezzenberger, 2000, S. 233, 244 ff; H.F. Müller, Konzern 2006, 725, 728 ff; abl. Hüffer, aaO (Fn. 47), $\$ 147$ AktG Rdn.2a; Koppensteiner, aaO (Fn. 38), $\$ 317$ AktG Rdn. 35. 
AktG konsequent aufgegriffen und konzerndimensional gewährt. Insgesamt sind die Vorgaben des kodifizierten Aktienkonzernrechts als effektiv sanktionierte Pflichtenordnung zu begreifen, mit der auf die rechtlich abgesicherte oder faktisch bestehende, konzernweite Leitungsmacht der Verwaltungsmitglieder der Obergesellschaft reagiert wird.

Für die hier primär untersuchte $\mathrm{GmbH}$ ist zwar im Ergebnis gesichert, dass das auf $Z$ weckbindung und mitgliedschaftlicher Treupflicht beruhende, strikte Schädigungsverbot zum Schutz der abhängigen, mehrgliedrigen $\mathrm{GmbH}^{81}$ auch von einzelnen Gesellschaftern klageweise durchgesetzt werden kann. ${ }^{82}$ Nicht ausdiskutiert ist aber, ob die bestehende Verantwortlichkeit auch die handelnden Organe des herrschenden Unternehmens erreicht. ${ }^{83}$ Der Hinweis auf die im Ausgangspunkt mitgliedschaftlichen Wurzeln des GmbH-rechtlichen Schädigungsverbots ${ }^{84}$ springt freilich schon deshalb zu kurz, weil auch

81 BGHZ 65, 15, 18 - „ITT“; BGH, NJW 1980, 231, 232 f - „Gervais“; BGHZ 95, 330, 340 - „Autokran“; Zöllner, aaO (Fn. 10), SchlAnhKonzernR Rdn. 77 ff; CAsPER, in: Ulmer/Habersack/Winter, GmbHG, Bd. 3, 2008, Anh. \$77 Rdn. 73; Scholz/EmMERICH, aaO (Fn.37); Anh. $\$ 13$ GmbHG Rdn.67ff; Liebscher, aaO (Fn. 5), Anh. $\$ 13$ GmbHG Rdn. 338 ff; Lutter, aaO (Fn. 5), Anh. $\$ 13$ GmbHG Rdn. 21; Habersack, aaO (Fn. 8), $\$ 318$ Rdn. 5, 24; EmMerich/Habersack, aaO (Fn. 13), $\$ 30$ III 1, S. 471 f; Raiser/Veil, aaO (Fn. 62), \53 Rdn. 49; K. Sснмidt, Gesellschaftsrecht, 4. Aufl., 2002, \39 III 2 b, S. 1220 ff; M. WINTER, Mitgliedschaftliche Treubindungen im GmbH-Recht, 1988, S. 113 ff; Wimmer-LeONHARDT, Konzernhaftungsrecht, 2004, S. 157 ff; dogmatisch, nicht aber im Ergebnis abweichend Flume, Allgemeiner Teil, Bd.1/2, 1983, \8 I, S. 270; DERs., ZIP 2006, 161, 165.

82 Für actio pro socio/actio pro societate insoweit BGHZ 65, 15, 19, 21 - „ITT“; Scholz/ Emmerich, aaO (Fn. 37); Anh. $\$ 13$ GmbHG Rdn. 87; Liebscher, aaO (Fn. 5), Anh. $\$ 13$ GmbHG Rdn. 430; Decher, Münchener Handbuch des Gesellschaftsrechts, Band 3, 3. Aufl., 2009, \$68 Rdn. 19; M. Winter, aaO (Fn. 81), S. 307 ff; Limmer, Die Haftungsverfassung des faktischen GmbH-Konzerns, 1992, S. $138 \mathrm{ff}$, eingehend ZöLlNER, ZGR 1988, 392, 408. Für Analogie zu $\$ \$ 309$ Abs. 4, 317 Abs. 4, 318 Abs. 4, 323 Abs. 1 Satz 2 AktG Casper, aaO (Fn. 81), Anh. $\$ 77$ GmbHG Rdn. 87; Lutter, aaO (Fn. 5), Anh. $\ 13$ GmbHG Rdn. 26; Habersack, aaO (Fn. 8), \$318 Rdn. 32; auch Assmann, FS 100 Jahre GmbHG, 1992, S. 657, $681 \mathrm{f}$.

83 Befürwortend z. B. Altmeppen, aaO (Fn. 28), S. 78 ff, 84 ff; Koppensteiner, aaO (Fn. 28), Anh. \52 GmbHG Rdn. 75; Altmeppen, aaO (Fn. 5), \$13 GmbHG Rdn. 102, Anh. $\$ 13$ GmbHG Rdn. 159; Jungkurth, aaO (Fn.62), S. 188 ff, Altmeppen, ZIP 2009, 49, 53 ff; erwägend Mülbert/Leuschner, NZG 2009, 281, 284; abl. Liebscher, aaO (Fn. 5), Anh. $\$ 13$ GmbHG Rdn.424; Habersack, aaO (Fn. 8), $\$ 318$ Rdn. 30 Fn. 81; Zöllner, aaO (Fn. 10), SchlAnhKonzernR Rdn. 109; speziell für die hier diskutierte Konstellation auch S. SchneIder, FS Uwe H. Schneider, S. 1177, 1194.

84 Diese sind auch nicht gekappt, wenn die Haftung für eine Verletzung des Schädigungsverbots auf eine faktische Organstellung des herrschenden Gesellschafters gestützt wird, so v. a. Wilhelm, Rechtsform und Haftung bei der juristischen Person, 1981, S. 253, 326, 352 ff; Stein, Das faktische Organ, 1984, S. 155, 183 ff; U. Schneider, ZGR 1980, 511, 534; Schulze-Osterloh, ZGR 1983, 123, 158 f; Wilhelm, DB 1986, 2113, 2117 ff; Altmeppen, ZIP 2001, 1553, 1562; Burgard, ZIP 2002, 827, 839, weil 
die aktienrechtliche Lösung darauf beruht, dass neben den - worauf auch immer gestützten Bindungen des herrschenden Gesellschafters ${ }^{85}$ - ein paralleles Pflichtenprogramm seiner Organe begründet wird. Gegenüber einer Verbreiterung des maßgeblichen, materiellen Schutzgedankens kann auch nicht eingewendet werden, der GmbH-rechtliche Lösungsansatz habe sich von den S $291 \mathrm{ff}$ AktG weitgehend „emanzipiert" ${ }^{86}$ weil auch die auf allgemeine verbandsrechtliche Prinzipien gestützte Lösung des GmbH-Rechts gemessen an ihrem telos unvollständig bliebe, wären die leitungsmächtigen Verwaltungsmitglieder der Obergesellschaft nicht in die sanktionsbewehrte Pflichtenbindung einbezogen. ${ }^{87}$

\section{b) Existenzvernichtungshaftung auch der Leitungsorgane als konsequentes Fortschreiben des normativen Prinzips}

Während das Pflichtenprogramm der $\int \$ 311 \mathrm{ff}$ BGB nach ganz überwiegender Meinung sowohl dem Minderheiten- als auch dem Gläubigerschutz dient, ${ }^{88}$ gewährleistet das mitgliedschaftsbasierte Schädigungsverbot des $\mathrm{GmbH}$ Rechts die Gläubigerinteressen nur als Reflex des Gesellschafterschutzes. Begreift man die Grundsätze der Existenzvernichtungshaftung als das neben den Kapitalerhaltungsgrundsätzen originär zum Schutz der Gläubigerbelange berufene und hierfür hinreichende Rechtsinstitut, ${ }^{89}$ darf dadurch nicht verdeckt werden, dass auch insoweit die genannten Gründe für eine Erstreckung der sanktionierten Pflichtenbindung auf die konzernweit Leitungsmacht aus-

auch deren Grundlage in der beteiligungsvermittelten Leitungsmacht liegt. Vgl. auch Flume, ZIP 1996, 161, der das Schädigungsverbot als unmittelbaren Ausfluss der Mitgliedschaftsrechte der benachteiligten Gesellschafter verankern möchte, was aber ersichtlich auch den zu begrenzenden, beteiligungsbasierten Einfluss der Mehrheit voraussetzt.

85 Die h. M. geht im Einklang mit der Sicht des historischen Gesetzgebers davon aus, dass das Schädigungsverbot des $₫ 311$ Abs. 1 AktG auch im Hinblick auf die Schutzinteressen der Aktionärsminderheit pflichtenbegründenden wirkt, was im Lichte der auch in der AG zwischenzeitlich ganz überwiegend bejahten, mitgliedschaftlichen Bindungen nicht überzeugt, eingehend Tröger, aaO (Fn. 76), S. $163 \mathrm{ff}, 210 \mathrm{ff}$.

86 So Liebscher, aaO (Fn. 5), Anh. $\$ 13$ GmbHG Rdn. 424.

$87 \mathrm{Zu}$ den Sachgründen o. III.

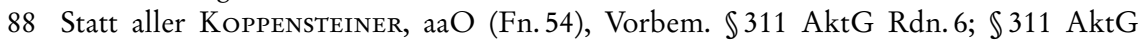
Rdn. 1; Altmeppen, aaO (Fn. 38), \$311 AktG Rdn. 3 ff; Habersack, aaO (Fn. 8), $\$ 311$ Rdn. 1; VetTer, in: K. Schmidt/Lutter, AktG, Bd.2, 2. Aufl., 2010, \$311 Rdn. 3.

89 Anders diejenigen, nach denen die Haftung für die Verletzung des Schädigungsverbots im GmbH-Recht auf einer analogen Anwendung des $₫ 317$ Abs. 1 AktG beruhen - und somit auch fahrlässige Beeinträchtigungen der Gläubigerinteressen sanktionieren soll, zuletzt z. B. Wilhelm, Kapitalgesellschaftsrecht, 2. Aufl., 2009, Rdn. 1320; Altmeppen, aaO (Fn. 5), Anh. $\mathbb{1} 13$ GmbHG Rdn. 156 ff. Zuvor auch Kropff, FS Kastner, 1992, S. 279, 296 ff; KropfF, FS Semler, 1993, S. 517, 536 ff. 
übenden Mitglieder der Verwaltung der Obergesellschaft sprechen. Der entscheidende Gedanke liegt darin, dass die angestrebte Internalisierung der gläubigerbenachteiligenden Handlungsfolgen über die Schadensersatzhaftung ganz und gar unabhängig davon wünschenswert ist, welche konkrete, im Gläubigerinteresse bestehende Pflicht verletzt wurde. Wird das relevante Pflichtenprogramm im Rahmen des $\ 826$ BGB über die Grundsätze des Existenzvernichtungsverbots umrissen, kann die Konsequenz folglich nur darin liegen, auch die Organwalter des herrschenden Unternehmens in die haftungsbewährte Bindung an die Gläubigerbelange einzubeziehen.

\section{Konsequenzen für die Passivlegitimation bei der Existenzvernichtungshaftung}

Aus dem Vorgesagten ist in der Tat die Konsequenz zu ziehen, die Mitglieder der Verwaltung des herrschenden Unternehmens selbst und unmittelbar nach $\$ 826$ BGB für die von Ihnen veranlasste Vernichtung der Existenz der abhängigen $\mathrm{GmbH}$ haften zu lassen. Mit anderen Worten, es bestehen parallel zu den Pflichten der Gesellschafter solche ihrer Organe, deren Verletzung ebenfalls zu einer Sonderdeliktshaftung führen. Demgegenüber sind anderweitige Versuche, zu der intendierten Haftung auch der Verwaltungsorgane zu gelangen abzulehnen. Umgekehrt hält sich die hier befürwortete Haftung nach $\$ 826$ BGB im Rahmen der insoweit maßgeblichen Dogmatik, die es grundsätzlich erlaubt, befriedigende und verbandsrechtlichen Spezifika Rechnung tragende Ergebnisse zu erzielen.

\section{Scheinlösung über eine Binnenhaftung nach $\int 823$ Abs. 2 BGB i. V.m. $\int 266 S t G B$}

Auch wenn der Meinungsstand zu der Frage, nach welchen Kriterien sich generell die Feststellung eines haftungsauslösenden Verstoßes gegen ein Schutzgesetz bemisst, durchaus uneinheitlich ist, ${ }^{90}$ wird der verbandsrechtlich erforderliche Schutz durch das zentrale Abstellen auf $\$ 823$ Abs. 2 BGB i. V. m. $\$ 266$ StGB ein Stückweit in einem sachfremden Kontext entwickelt und par-

90 Neben der alleinigen Maßgeblichkeit der für das Schutzgesetz geltenden Grundsätze (RGZ 118, 312, 315; RGZ 166, 40, 46; Wagner, aaO (Fn. 5), \823 BGB Rdn.361; Baumann, AcP 155 (1956) 511 ff; Medicus, SchuldR II, 15. Aufl., 2010, Rdn. 1324; Spindler, aaO (Fn. 6), $\$ 823$ BGB Rdn. 163; Deutsch/Ahrens, Deliktsrecht, 5. Aufl., 2009, Rdn. 290) wird dafür plädiert, die Wertungen des betreffenden Rechtsgebiets in diejenigen des Haftungsrechts einfließen zu lassen (SPIскноғғ, in: Soergel, BGB, Bd. 12, 13. Aufl., 2005, $\$ 823$ Rdn. 206). Ausschließlich auf den Maßstab des allgemeinen zivilrechtlichen Haftungsrechts abgestellt wird hingegen nach BECKER, Das Recht der unerlaubten Handlungen, 1976, S. 461. 
tiell an die Voraussetzungen staatlichen Strafens gekoppelt. Wichtiger ist im hier interessierenden Kontext aber, dass die „Blankettvorschrift“ des $\$ 266$ StGB eine verbandsrechtlich begründete Pflicht des Vorstands voraus setzt und daher im Unrechtskern zivilrechtsakzessorisch ist. ${ }^{91}$ Wenn man aber prinzipiell bereit ist, den Geschäftsleiterpflichten schadensersatzrechtliche Relevanz gegenüber der beeinträchtigten Untergesellschaft zuzubilligen, sollte man diese Erkenntnis nicht über den Umweg eines strafrechtlichen Schutzgesetzes umsetzen. Überzeugender ist es, sich klar zu Schädigungsverboten im Verbandsrecht zu bekennen, die auch zugunsten der Konzerntöchter bestehen und im Verletzungsfall unmittelbar durch die einschlägigen verbandsrechtlichen Institute sanktioniert werden ${ }^{92}-\mathrm{zu}$ denen nach der hier vertretenen Konzeption auch die Sonderdeliktshaftung nach $\$ 826$ BGB wegen Existenzvernichtung gehört.

\section{Scheinlösung über die Haftung als Tatteilnehmer, $\int 830$ Abs. 1, Abs. 2 BGB}

Ebenso wenig überzeugt eine andere dogmatische Hilfskonstruktion, die von Teilen der Literatur befürwortet wird, die für eine Haftung von Organen als Mittäter ( $\ 830$ Abs. 1 BGB) oder zumindest Teilnehmer ( $\$ 830$ Abs. 2 BGB) des die Existenzvernichtung herbeiführenden Gesellschafters plädieren. ${ }^{93} \mathrm{Da}-$ bei haben die Protagonisten dieser Literaturmeinung freilich zumeist die Einbeziehung des Geschäftsführers der insolventen $\mathrm{GmbH}$ in das - neben die Haftung nach $\$ \$ 43$ Abs. 2, $64 \mathrm{GmbHG}$ tretende - deliktsrechtliche Verantwortlichkeitssystem im Auge, nicht aber die Haftung der Verwaltungsmitglieder eines an der $\mathrm{GmbH}$ beteiligten Verbands. Für diese überzeugt denn auch eine Begründung der Verantwortlichkeit als Tatbeteiligte über $\ 830$ BGB nicht. ${ }^{94}$ Der handelnde Organwalter repräsentiert die juristische Person, deren Haftung beruht auf der Zurechnung seines Handelns gemäß $\$ 31$ BGB. Es bedarf keiner vertieften Auseinandersetzung um die zutreffende dogmatische Einordnung der Organhaftung oder gar des „Wesens“ der juristischen Per-

91 Vgl. BGH ZIP 2001, 1874, 1876f; BGHZ 150, 61, 63, 68. Auch schon Fleck, FS 100 Jahre GmbHG, 1992, S. 391, 298.

92 Krit. gegenüber dem Strafrechtsimport auch SсHÖN, ZHR 168 (2004) 268, 285 f; HaAs, ZIP 2006, 1373, 1380 f; GrigoleIT, Gesellschafterhaftung für interne Einflussnahmen im Recht der GmbH, 2006, S. 216 ff.

93 Oechsler, aaO (Fn. 6), $\$ 826$ BGB Rdn. 325b; Scholz/U. SchneIder, aaO (Fn. 61), $\$ 43$ GmbHG Rdn. 287a; Lutter, aaO (Fn. 5), \13 GmbHG Rdn. 46; Altmeppen, aaO (Fn. 5), $₫ 13$ GmbHG Rdn. 102, \43 Rdn. 86; Kölbl, BB 2009, 1194, 1198;Weller, ZIP 2007, 1681, 1687; Gehrlein, WM 2008, 761, 764; Schaefer/SteinmetZ, WM 2007, 2265, 2271; Habersack, ZGR 2008, 533, 546; deutlich enger VetTER, BB 2007, 1965, 1968 f; allgemein auch schon BGHZ 105, 121, $133 \mathrm{f}$ („Kerkerbachbahn“).

94 Eingehend zu dieser Konstellation und i.Erg. wie hier ablehnend jetzt auch S. ScHNEIDER, FS Uwe H. Schneider, S. 1177, 1183 ff; DERS., GmbHR 2011, 685, 687 ff. 
son, ${ }^{95}$ um zu erkennen, dass hier ein fiktives Zusammenwirken des handelnden Organs mit der von ihm repräsentierten Gesellschaft konstruiert wird, ${ }^{96}$ um das gewünschte Ergebnis zu erreichen. ${ }^{97}$

Die fehlende Überzeugungskraft des Konstrukts einer Mittäter- oder Teilnehmerbeziehung des Organs zu seiner Gesellschaft wird im Übrigen in der vergleichbaren Konstellation bei der Emittentenhaftung nach $\$ \$ 37 \mathrm{~b}, 37 \mathrm{c}$ WpHG durchaus zutreffend erkannt, wo die herrschende Meinung eine Eigenhaftung der Organe des Emittenten zu recht ablehnt. ${ }^{98}$ Neben dem auch in diesem Zusammenhang vorgebrachten Argument der Künstlichkeit der Konstruktion scheidet eine (Mit-)Täterschaft des Organs nach ganz überwiegender Auffassung in der Sache aus, da die Verantwortung für die zutreffende und vollständige Kapitalmarktinformation ausschließlich den Emittenten treffe, ${ }^{99}$ also nur dieser die den Haftungstatbestand auslösenden Voraussetzungen in eigener Person zu erfüllen in der Lage ist. Folglich fehlt es dem handelnden Organ zur Verwirklichung dieses Sonderdelikts an der notwendigen Täterqualität.

Daraus lässt sich für die hier interessierende Fragestellung folgern, dass eine Tatbeteiligung der Organe der Obergesellschaft nur in Betracht kommt, soweit sich das Verbot der Existenzvernichtung auch an diese im Sinne einer originären Außenpflicht richtet, mit anderen Worten, wenn sie eine Bestandsverantwortung auch gegenüber der Tochtergesellschaft selbst trifft. Bejaht man diese, wofür die hier eingehend dargelegten Gründe sprechen, bestehen

$95 \mathrm{Zu}$ der Kontroverse zwischen Vertreter- und Organtheorie, die letztlich in dem vor allem zwischen v. SAvigny und v. Gierke ausgefochtenen Streit um das zutreffende Verständnis der Rechtssubjektivität der juristischen Person wurzelt (zu diesem HeNKEL, Zur Theorie der Juristischen Person im 19. Jahrhundert, 1972, S. 71 ff, 192 ff; FLUME, FS Franz Wieacker, 1978, S. 340; KLEIndiek, Deliktshaftung und juristische Person, 1997, S. 153 ff) nur MARTineK, Repräsentantenhaftung, 1979, S. 25 ff.

$96 \mathrm{Zu}$ den diesbezüglichen Voraussetzungen gerade im Hinblick auf die hier in Rede stehende Konstellation S. SchneIder, FS Uwe H. Schneider, S. 1177, 1184 f; DERs., GmbHR 2011, 685, 686; auch FleisCHER, AG 2008, 265, 269.

97 Zutr. demgegenüber S. SchneIder, FS Uwe H. Schneider, S. 1177, 1186; DERs., GmbHR 2011, 685, 687; der auf die Wertungsgebundenheit der Annahme entsprechender Teilnahmebeziehungen hinweist.

98 Casper, Kölner Komm. z. KapMuG, 2008, \$§37b, 37c WpHG Rdn. 83; Möllers/ Leisch, Kölner Komm. z. WpHG, 2007, $\$ \$ 37$ b, c Rdn. 80; Zimmer/Grotheer, in Schwark/Zimmer (Hrsg.), KMRK, 4. Aufl., 2010, \$\$ 37b, 37c WpHG Rdn. 130; BucKHees, Kapitalmarktrecht, 4. Aufl., 2010, Rdn. 324; Fleischer, AG 2008, 265, 271; Hellgardt, Kapitalmarktdeliktsrecht, 2008, S. 415 ff; Dogan, Ad-hoc-Publizitätshaftung, 2005, S. 218; für eine persönliche Haftung der Organe neben dem Emittenten nach \$S 37b, c WpHG, 830 Abs. 2 BGB, Rieckers, BB 2002, 1213, 1220; SchWARK, EWiR 2001, 1049, 1050.

99 Möllers/Leisch, aaO (Fn. 98), $\$ \int 37$ b, c WpHG Rdn. 80; Sethe, in: Assmann/U. Schneider, WpHG, 5. Aufl., 2009, $\mathbb{S}$ 37b, 37c Rdn. 133; Buck-HeEb, aaO (Fn. 98), Rdn. 324; Maier-Reimer/Webering, WM 2002, 1857, 1864; Casper, BKR 2005, 83, 86. 
keine erkennbaren Hindernisse, die Verletzung der Pflicht im Rahmen der direkten Anwendung des $₫ 826 \mathrm{BGB}$ zu sanktionieren. Anders als im Rahmen der bewusst eng gefassten $\$ \$ 37 \mathrm{~b}, 37 \mathrm{c} \mathrm{WpHG}{ }^{100}$ kann der Anspruchsgrundlage als solcher prinzipiell keine Beschränkung der Passivlegitimation entnommen werden. Gegenüber einer im hier vertretenen Sinne normativ fundierten, besonderen Pflichtenbindung der Leitungsorgane verfängt auch der Einwand nicht, im GmbH-Recht fehle es an einer allgemeinen, deliktsrechtlichen Verantwortlichkeit für schädigende Einflussnahmen, wie sie im Aktienrecht nach $\$ 117$ AktG bestehe. ${ }^{101}$ Es geht materiell eben gerade nicht um die simple Subsumtion verbandsrechtlicher Sachverhalte unter Haftungsnormen, die eine Verletzung von Jedermannspflichten sanktionieren. Vielmehr wird das Anliegen verfolgt, eine besondere Bestandsverantwortung durch eine spezifisch kapitalgesellschaftsrechtliche Entfaltung der qualifizierten deliktsrechtlichen Generalklausel zu entwickeln. Ist man aber bereit, die Leitungsorgane in diesem Rahmen in die Pflicht zu nehmen, sollte man sich die Hose nicht mit der Kneifzange anziehen, wenn man sie auch einfach überstreifen kann.

\section{Vorzugswürdigkeit des Fortschreibens der gesellschaftsrechtlichen Verantwortlichkeit}

Insgesamt erscheint daher vorzugswürdig, die normativen Desiderate im Rahmen einer Ausweitung der Sonderdeliktshaftung auf die Verwaltungsmitglieder der Obergesellschaft umzusetzen. Dogmatisch wird hiermit keinesfalls Neuland betreten, sondern lediglich ein Weg beschritten, der bereits vorgezeichnet ist und zu adäquaten Ergebnissen führt.

\section{a) Deliktische Haftung der Geschäftsführer und Vorstände nach $\int 826 B G B$ jenseits von Existenzvernichtungstatbeständen}

Schon lange vor Trihotel und der Ansiedlung der Existenzvernichtungshaftung in der qualifizierten deliktsrechtlichen Generalklausel war die Möglichkeit einer Organaußenhaftung aus $\$ 826$ BGB in einer Vielzahl von Fallgestal-

100 Die Restriktionen im Rahmen der $\int \$ 37 b, 37 \mathrm{c}$ WpHG sollten durch die geplante Organaußenhaftung in $\$ 37$ a Abs. 2 WpHG i. d. F. des gescheiterten Kapitalmarktinformationshaftungsgesetz (KapInHaG) überwunden werden, das auf einen Vorschlag der Regierungskommission Corporate Governance (BAums, Bericht der Regierungskommission „Corporate Governance“, BT-Drs. 14/7515, Rdn. 186) und auf einen Beschluss des 64. DJT (Beschlüsse des 64. DJT, NJW 2002, 3073, 3082 Nr. 1.11) einging, der auf der Grundlage des Gutachtens (Fleischer, Gutachten F für den 64. DJT, S. 99 ff) gefasst worden war. De lege ferenda auch SPINDLER, DStR 2002, 1576, 1580.

101 So aber S. Schneider, FS Uwe H. Schneider, S. 1177, 1193; DERs., GmbHR 2011, 685, 690. 
tungen im Grundsatz anerkannt. Die hierzu bestehende Kasuistik ist umfangreich; etablierte Fallgruppen lassen sich aber anhand der Schutzrichtung der verletzten Pflichten unterscheiden. Als Gläubiger eines Ersatzanspruches kommen vor allem drei Gruppen in Betracht, deren Interessen durch Pflichten im Sinne des $₫ 826$ BGB gewährleistet werden sollen: die das Organ anstellende Gesellschaft, ${ }^{102}$ deren Gesellschafter ${ }^{103}$ sowie außenstehende Gläubiger.

Die größte Anzahl höchstrichterlicher Entscheidungen ist zu von Gläubigern der Gesellschaft geltend gemachten Ansprüchen - mithin zu der für die vorliegende Untersuchung relevanten Konstellation-erfolgt. ${ }^{104}$ Das Spektrum der als sittenwidrig erachteten Schädigungshandlungen der Geschäftsleitung reicht hier von vorsätzlicher Insolvenzverschleppung, ${ }^{105}$ der Verletzung von Aufklärungspflichten bei der Vermittlung risikoreicher Anlagegeschäfte ${ }^{106}$ über grob unrichtige Information des Kapitalmarktes ${ }^{107}$ bis hin zur bewusst wahrheits-

102 Im Verhältnis zur Gesellschaft selbst kann eine Verantwortlichkeit aus $₫ 826$ BGB neben die Ansprüche aus $\$ \mathbb{S} 43 \mathrm{GmbHG}, 93 \mathrm{AktG}$ treten. Hierbei gilt es jedoch zu beachten, dass nicht jedweder Verstoß gegen Organpflichten per se den Tatbestand des $\$ 826$ erfüllt, da die Schwelle zur Sittenwidrigkeit hoch anzusiedeln ist. Selbst im Falle eigennützigen Verhaltens des Organs soll dies erst bei einer groben Missachtung des Mindestmaßes an Loyalität und Rücksichtnahme anzunehmen sein, vgl. BGH ZIP 1390, 1394 f; Scholz/U. Schneider, aaO (Fn. 61), \$43 GmbHG Rdn. 286.

103 Was eine Ersatzpflicht wegen sittenwidrigen Verhaltens der Geschäftsleiter gegenüber den Gesellschaftern (vgl. hierzu Hopt, aaO (Fn. 62), \$93 AktG Rdn. 480 ff; Zöllner/ Noack, aaO (Fn. 10), $\$ 43$ GmbHG Rdn. 64; Paefgen, in: Ulmer/Habersack/Winter, GmbHG, 2005, $\$ 43$ Rdn. 186) anbelangt, so wird dies für die $\mathrm{GmbH}$ beispielsweise im Falle nicht von den Gesellschaftern genehmigter Veräußerungen von Gesellschaftsanteilen angenommen (HAAS/MüLLER, GmbHR 2004, 1169, 1173 f) während der Vorstand einer AG eine Haftung aus $\$ 826$ BGB besonders bei sittenwidriger Durchführung von Kapitalmaßnahmen fürchten muss, so z. B. bei Vornahme einer Kapitalerhöhung, die alleine dem Zweck der Insolvenzverschleppung dient (ggü. den Neuaktionären) BGHZ 96, 231, 243.; SPINDleR, aaO (Fn. 62), \$93 AktG Rdn. 280; DERs., in: Spindler/ Stilz, AktG, Bd.1, 2. Aufl., 2010, $\$ 116$ Rdn. 194 oder für den Fall eines teilweisen Bezugsrechtsausschlusses, um die Vorherrschaft der Mehrheits- zulasten der Minderheitsaktionäre sicherzustellen, vgl. RGZ 107, 72, $74 \mathrm{f}$.

104 Einen Überblick über die verschiedenen Fallgruppen gewährend SANDberger, Die Außenhaftung des GmbH-Geschäftsführers, 1997, S. $223 \mathrm{ff}$.

105 BGHZ 108, 134, 142; BGH ZIP 2008, 361, 362; Kleindiek, aaO (Fn.61); \$43 GmbHG Rdn. 72; Zöllner/NoAck, aaO (Fn. 10), $₫ 43$ GmbHG Rdn. 86.

106 BGH ZIP 2005, 158; Altmeppen, aaO (Fn. 5), \$43 GmbHG Rdn. 84 f; Zöllner/NoAcK, aaO (Fn. 10), $₫ 43$ GmbHG Rdn. 86.

107 BGHZ 160, 134 - „Infomatec I“; BGHZ 160, 149 - „Infomatec II“; BGH NJW 2005, 2450 - „EM.TV“; BGH ZIP 2007, 681 - „Comroad I“; BGH ZIP 2007, 679 - „Comroad II“; BGH ZIP 2007, 326 - „Comroad III“; BGH ZIP 2007, 1560 - „Comroad IV“; BGH ZIP 2007, 1564 - „Comroad V“; BGH ZIP 2008, 407 - „Comroad VI“; BGH ZIP 1008, 410 - „Comroad VII“; BGH ZIP 2008, 829 - „Comroad VIII“; Dies bestätigt die oben V.2. gemachten Ausführungen: Aufgrund der vorsätzlich unwahren adhoc-Mitteilung wird die Schwelle des $\$ 826$ BGB überschritten und die Eigenhaftung 
widrigen Täuschung über die Leistungsfähigkeit der Gesellschaft. ${ }^{108}$ Der zentrale Gesichtspunkt liegt für die hier angestellten Überlegungen allein darin, dass $\ 826$ BGB nach gängiger Lesart eine gläubigerschützende Dimension dergestalt erlangen kann, dass die Leitungsorgane aufgrund einer eigenen Pflichtenbindung neben der Gesellschaft den Gläubigern haften, wenn sich die Haftung des Verbands allein als insuffizient erweist. Lässt sich demnach die eigene Bestandsverantwortung der Organwalter auf der Grundlage der hier angestellten Überlegungen materiell begründen, ${ }^{109}$ steht einer dogmatischen Umsetzung der Erkenntnisse im Rahmen des $₫ 826$ BGB kein grundsätzlicher Einwand entgegen.

\section{b) Sachgerechte Ergebnisse auf der Grundlage der Sonderdeliktshaftung}

Schließlich ist der bisweilen geäußerten Kritik eine Absage zu erteilen, nach der eine Haftung in den Bahnen des allgemeinen Deliktsrechts spezifisch gesellschaftsrechtliche Fragestellungen nicht ausreichend differenziert beantworten könne. ${ }^{110}$ Gerade die Eigenarten der gesellschaftsrechtlichen Problemlagen spiegeln sich im Rahmen der Verantwortlichkeit aus $\$ 826$ BGB in sämtlichen erwähnten Konstellationen wider. Als Anknüpfungspunkt für das Einfließen organisationsrechtlicher Wertungen dient dabei das Tatbestandsmerkmal der Sittenwidrigkeit. ${ }^{111}$ Im verbandsrechtlichen Kontext kommt man zwar mit dem allgemeinen Verständnis der Sittenwidrigkeit als Verstoß gegen das Rechts- und Sittlichkeitsbewusstsein der Staatsbürger ${ }^{112}$ nicht weit. Anzunehmen, dass das potentiell haftungsauslösende Verhalten mit dem Anstandsgefühl aller billig und gerecht Denkenden ${ }^{113}$ unvereinbar sein müsse, ist insofern verfehlt, als der Sittenverstoß keinesfalls eine Übertretung von allgemeinen „Moralvorstellungen“ oder einen Kontext zu „sexuell anstößigem Verhalten" aufzuweisen braucht. ${ }^{114}$ Eine allgemeingültige Formulierung sittenwidrigen Verhaltens im Rechtsverkehr ist daher schlechterdings nicht mög-

des Vorstandes (also nicht als bloßer Teilnehmer) auf der Grundlage seiner eigenen, originären Pflichten möglich. Dass die Vorstandspflichten nicht parallel zu denjenigen der Gesellschaft (des Emittenten) verlaufen, ist den positivrechtlich begründeten Besonderheiten der Emittentenhaftung nach $\$ \$ 37 \mathrm{~b}, 37 \mathrm{c}$ WpHG geschuldet.

108 BGHZ 87, 27, 34; BGH GmbHR 1994, 464; OLG Naumburg, GmbHR 2001, 974; Paefgen, aaO (Fn. 103), $\ 43$ GmbHG Rdn. 222; Kleindiek, aaO (Fn.61), $₫ 43$ GmbHG Rdn. 72; Zöllner/NoAcK, aaO (Fn. 10), $\$ 43$ GmbHG Rdn. 86.

109 Vgl. oben III. und IV.

110 Altmeppen, ZIP 2001, 1837, 1842.

111 RaIser, FS Marcus Lutter, 2000, S. 637, 638.

112 Bzw. eines speziellen Verkehrskreises, vgl. BGHZ 10, 228, 232; BGHZ 17, 327, 332.

113 St. Rspr. seit RGZ 80, 219, 221.

114 So auch WAGNER, FS Claus-Wilhelm Canaris, S. 473, 495; nach WAGNER, aaO (Fn. 5), \826 BGB Rdn. 9 handelt es sich hierbei vielmehr um eine „klassische Leerformel“, die für sich betrachtet nichts auszusagen vermag. 
lich; dieses ist vielmehr aus dem jeweiligen Sachgebiet heraus zu definieren. ${ }^{115}$ Im speziellen Fall der Missachtung oder Umgehung wesentlicher Institute des verbandsrechtlichen Gläubigerschutzes durch Gesellschafter oder Geschäftsleitung, vermag dies die Ersatzfähigkeit eines bloßen Vermögensschadens als nach den speziellen Wertungen des Rechtsgebiets sittenwidriger Schädigung nach $\ 826$ BGB zu rechtfertigen. So etwa wenn die Akteure der Gesellschaft Vermögen mit dem Zweck entziehen, einen Zugriff der Gläubiger zu verhindern, ${ }^{116}$ in Insolvenznähe auf Kosten der Gläubiger spekulieren u.ä.

\section{c) Eigenständige Sonderdeliktshaftung und Organhaftung}

Gegenüber der hier eingehend begründeten, normativen Verankerung einer selbständigen Sonderdeliktshaftung der Fremdorgane des GmbH-Gesellschafters springt eine Argumentation zu kurz, die deren unmittelbare Verantwortlichkeit vor allem deshalb ablehnt, weil weder die Verhaltens- noch die Verschuldenszurechnung nach $\$ 31$ BGB (analog) eine eigene Pflichtenbindung der Handelnden voraussetze. ${ }^{117} \mathrm{Z}$ war trifft es zu, dass die zur Begründung der Verantwortlichkeit des die GmbH-Anteile haltenden Verbands erforderliche Zurechnung des Handelns seiner Verwaltungsorgane nach h. M. keine eigene Pflichtenbindung dieser Personen voraussetzt. ${ }^{118}$ Entscheidend ist aber, dass eine zur persönlichen Verantwortlichkeit führende, eigene Pflichtenbindung der Organmitglieder von den Zurechnungsvoraussetzungen des $\$ 31$ BGB (analog) unabhängig ist. Mit anderen Worten, auch wenn die deliktische Eigenhaftung des Geschäftsführers, Vorstands etc. keine gedanklich zwingende Voraussetzung der Haftung der Anstellungskörperschaft nach $\$ \int 826,31$ BGB darstellt, ${ }^{119}$ kann eine solche dogmatisch-konstruktiv ohne Verwerfungen neben die Gesellschafterhaftung treten. Allein entscheidend bleibt somit, dass für die eigene Verantwortlichkeit der leitungsmächtigen Organmitglieder die dargelegten Wertungsgesichtspunkte sprechen.

\section{VI. Übertragbarkeit auf den Aktienkonzern}

Das Institut der Existenzvernichtungshaftung ist in der Judikatur ausschließlich als Reaktion auf Eingriffe in die wirtschaftliche Lebensfähigkeit einer

115 Wagner, FS Claus-Wilhelm Canaris, S. 473, 495.

116 BGH NJW 2005, 145, 146; BGHZ 151, 181.

117 So S. Schneider, FS Uwe H. Schneider, S. 1177, $1181 \mathrm{ff}$.

118 Reuter, Münchner Komm. z. BGB, Bd.1/1, 5. Aufl., 2006, \31 Rdn. 31; eingehend Kleindiek, Deliktshaftung und juristische Person, 1997, S. 238 ff, 320 ff, 356.

119 So aber das Konzept einer unselbständigen, abgeleiteten Haftung der juristischen Person, hierfür z. B. BrüggemeIER, AcP 191 (1991) 33, 65 f. 
(Einpersonen-) $\mathrm{GmbH}$ entwickelt worden. Es ist daher zu hinterfragen, ob ein Transfer der oben dargestellten Grundsätze von der $\mathrm{GmbH}$ auf die abhängige Aktiengesellschaft möglich ist, ob also die hier befürwortete Verantwortlichkeit auch die Organe der Obergesellschaft treffen können, die eine Aktiengesellschaft beherrscht. Unabhängig vom Kreis der Haftenden wurde zum Teil die (rechtsform-)spezifische Begründung der Verantwortlichkeit im Rahmen des qualifizierten faktischen Konzerns und der Durchgriffshaftung als mit dem Aktien(konzern)recht inkompatibel angesehen. ${ }^{120}$ Mit vergleichbarer Argumentation wird die Übertragbarkeit der Grundsätze auch nach der Neuausrichtung des Haftungskonzepts bereits im Ausgangspunkt bestritten. ${ }^{121}$ In der Tat entspricht es der hier vertretenen Sicht, dass die jüngst erfolgte Ansiedlung des Haftungskonzepts in $\$ 826 \mathrm{BGB}^{122}$ keine Vorentscheidung über den Adressatenkreis der Sonderdeliktshaftung enthält. ${ }^{123}$ Insofern lässt sich noch nicht aus der systematischen Stellung der Anspruchsgrundlage folgern, dass es sich bei der Haftung für Existenzvernichtung keinesfalls um ein Spezifikum des $\mathrm{GmbH}-$ Rechts handeln könne. ${ }^{124}$ Es ist gerade unter Berücksichtigung der rechtsformspezifischen Besonderheiten des Verbandsrechts zu begründen, ob die Anknüpfung des Deliktsrechts an die jeweiligen Sozialpflichten tatsächlich als allgemeiner Grundsatz des Kapitalgesellschaftsrechts verstanden werden darf ${ }^{125}$ und deshalb mit einer verbreiteten Ansicht davon auszugehen ist, die Existenzvernichtungshaftung gelte auch im Recht der Aktiengesellschaft. ${ }^{126}$

Die komplexe Grundsatzfrage kann an dieser Stelle nicht adäquat erörtert werden. Im Hinblick auf den begrenzten Untersuchungsgegenstand dieser Abhandlung ist aber immerhin zu konstatieren, dass die spezifischen Gründe, die für eine Erstreckung der Verantwortlichkeit auf die Verwaltungsmitglieder der Obergesellschaft angeführt wurden, ${ }^{127}$ strukturell auch im Aktienrecht

120 Schürnbrand, ZHR 169 (2005), 35, 58; K. Schmidt, aаO (Fn. 72), 『31 IV.4.a), S. 964 ff; Müller, in: Spindler/Stilz, AktG, Bd.2, 1. Aufl., 2007, Vor $\ 311$ Rdn. 26; FETT, in: Bürgers/Körber, AktG, 2. Aufl., 2011, \$311 Rdn. 30; CAHN, ZIP 2001, 2159, 2160.

121 Habersack, aaO (Fn. 8), Anh. zu $₫ 317$ Rdn. 5; Müller, aaO (Fn. 120), Vor $\ 311$ AktG Rdn. 26; Krieger, aaO (Fn. 31), \$69 Rdn. 134.

122 BGHZ 173, $246 \mathrm{ff}$.

123 Schon oben I.

124 Es ist eine andere Frage, dass jedenfalls die praktische Bedeutung des Instituts im Recht der GmbH größer sein dürfte als im Aktienrecht, vgl. Fock, in: Spindler/Stilz, AktG, Bd.1, 2. Aufl., 2010, $\mathbb{1}$ Rdn. 64; Westermann, in: Bürgers/Körber, AktG, 2. Aufl., 2011, $\mathbb{1} 1 \mathrm{Rdn} .31$.

125 So Fock, aaO (Fn. 124), $\$ 1$ AktG Rdn. 64.

126 Dafür Fock, aaO (Fn. 124), $\$ 1$ AktG Rdn. 64.; Heıder, Münchener Komm. z. AktG, Bd.1, 3. Aufl., 2008, $\mathbb{1}$ Rdn. 85; Westermann, aaO (Fn.124), $\mathbb{1}$ AktG Rdn.31; Koppensteiner, aaO (Fn. 54), Anh. $\$ 318$ AktG Rdn. 63 ff.

127 Oben III. und IV. 
valide bleiben. Mit anderen Worten, sofern die Sonderdeliktshaftung auch zur Gewährleistung der Interessen der Gläubiger der abhängigen Aktiengesellschaft berufen ist, erstreckt sich der Kreis der Haftenden auch auf die Organe der Obergesellschaft.

Kein durchschlagender Einwand gegen diese Sicht erwächst dabei aus der Überlegung, dass eine unmittelbare Verantwortlichkeit der Leitungsorgane einer körperschaftlich verfassten Aktionärin auch nach Maßgabe des $\mathbb{} 117$ AktG in Betracht kommt. ${ }^{128}$ Zum einen wird ganz überwiegend die Anwendbarkeit des $₫ 826 \mathrm{BGB}$ neben $\ 117 \mathrm{AktG}$ bejaht. ${ }^{129}$ Zum anderen ist festzuhalten, dass sich an der materiell interessierenden, deliktsrechtlichen ${ }^{130}$ Pflichtenlage auch dann nichts änderte, wenn deren positivrechtliche Verankerung im Aktienrecht eine andere wäre.

\section{Thesen}

1. Die dogmatische Verortung der Haftung wegen existenzvernichtender Eingriffe in der qualifizierten deliktsrechtlichen Generalklausel des $\ 826$ BGB ändert nichts an ihren normativen Wurzeln im Verbandsrecht. Ausgehend von diesen Wertungsgrundlagen ist die Frage nach der zutreffenden Abgrenzung des Adressatenkreises der Sonderdeliktshaftung zu beantworten.

2. Die Agenturtheorie liefert ein analytisches Instrumentarium, mit dessen Hilfe die Notwendigkeit einer Verantwortlichkeit der Verwaltungsmitglieder der Obergesellschaft, die ihrer konzerndimensionalen Leitungsmacht entspricht, überpositiv begründet werden kann. Sie stellt ein Komplement zu der in der juristischen Literatur gängigen Beschreibung des „Konzernkonflikts" dar, auf dessen Grundlage die konfligierenden Interessen der Gläubiger der Untergesellschaft und der Anteilseigner der Obergesellschaft und ihrer Repräsentanten auf der Ebene der Geschäftsleitung analysiert werden können.

3. Die Binnenhaftung der Organwalter der Obergesellschaft gegenüber ihrer Anstellungskörperschaft führt zu Vollzugsdefiziten im Hinblick auf die wün-

128 Z. B. Spindler, aaO (Fn. 62), \117 AktG Rdn. 15; Kort, aaO (Fn. 62), \117 AktG Rdn. 132. Hierauf weist auch S. SchneIder, FS Uwe H. Schneider, S. 1177, 1192 zutreffend hin, der daraus aber primär ein Argument gegen eine entsprechende Haftung im GmbH-Recht ableitet (dazu schon oben V.2.).

129 BegrRegE bei Kropff, aaO (Fn.77), S.164; Spindler, aaO (Fn. 62), \117 AktG Rdn. 88; HüFfer, aaO (Fn. 47), $₫ 117$ AktG Rdn.14; Voigt, Haftung aus Einfluss auf die Aktiengesellschaft (\$S 117, 309, 317 AktG), 2004, S. 264.

130 Zu der ganz überwiegenden Qualifizierung des $\$ 117$ AktG als deliktsrechtlicher Haftungstatbestand, BGH NJW 1992, 3167, 3172; BGHZ 129, 136, 160 „Girmes“; SPINDLer, aaO (Fn. 62), $\mathbb{1 1 7}$ AktG Rdn. 4 f; Mertens, Kölner Komm. z. AktG, Bd. 2/2, 2 Aufl., 1996, $\$ 117$ Rdn. 9; Hommelhoff/Witt, in: K. Schmidt/Lutter (Hrsg.), AktG, Bd. 1, 2. Aufl., 2010, $\$ 117$ Rdn. 2. 
schenswerte Verantwortlichkeit, die auch durch die (analoge) Anwendung aktienrechtlicher Gläubigerverfolgungsrechte nicht voll aufgefangen werden.

4. Die $\mathbb{\$} 309$ Abs.2, 317 Abs. 3 AktG entsprechen dem institutionenökonomisch begründeten Gleichlauf von Haftungsverantwortung und konzerndimensionaler Leitungsmacht. Die konsequente Umsetzung der im Grundsatz auch für das GmbH-Recht maßgeblichen, materiellen Schutzüberlegungen gebietet eine Erstreckung der Existenzvernichtungshaftung auf die Leitungsorgane des herrschenden Unternehmens.

5. Die dogmatische Umsetzung der postulierten, normativen Ziele sollte über die Annahme einer eigenen Pflicht der Verwaltungsmitglieder erfolgen, deren vorsätzliche Verletzung unmittelbar zur Haftung nach $\$ 826$ führt. Im Rahmen einer verbandsrechtsspezifischen Konkretisierung der qualifizierten Generalklausel des Deliktsrechts lassen sich sachgerechte Ergebnisse erzielen. Alternativlösungen über eine Teilnehmerhaftung oder die haftungsrechtliche Instrumentalisierung von Straftatbeständen sind demgegenüber nicht zielführend.

6. Sofern das Institut des bestandsvernichtenden Eingriffs auch im Recht der Aktiengesellschaft Anwendung findet, gelten die hier entwickelten Grundsätze der Haftung der Verwaltungsmitglieder der Obergesellschaft auch bei erfassten Schädigungen der abhängigen AG. 